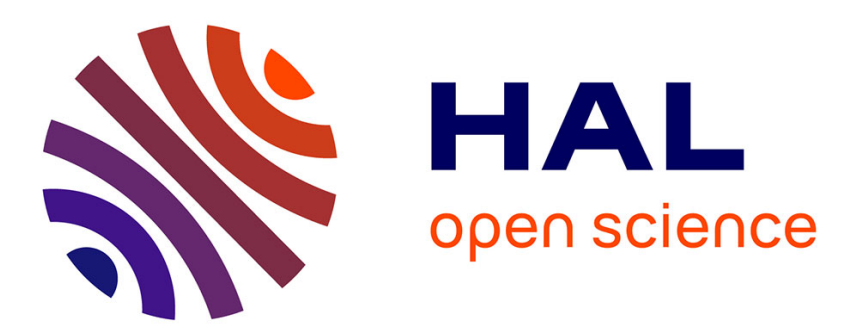

\title{
Conditions d'optimisation du traitement des matériaux métalliques par laser. I. Etablissement d'une méthodologie
}

\author{
J. Dietz, J. Merlin
}

\section{- To cite this version:}

J. Dietz, J. Merlin. Conditions d'optimisation du traitement des matériaux métalliques par laser.

I. Etablissement d'une méthodologie. Revue de Physique Appliquée, 1988, 23 (11), pp.1787-1805. 10.1051/rphysap:0198800230110178700 . jpa-00246009

\section{HAL Id: jpa-00246009 https://hal.science/jpa-00246009}

Submitted on 1 Jan 1988

HAL is a multi-disciplinary open access archive for the deposit and dissemination of scientific research documents, whether they are published or not. The documents may come from teaching and research institutions in France or abroad, or from public or private research centers.
L'archive ouverte pluridisciplinaire HAL, est destinée au dépôt et à la diffusion de documents scientifiques de niveau recherche, publiés ou non, émanant des établissements d'enseignement et de recherche français ou étrangers, des laboratoires publics ou privés. 


\title{
Conditions d'optimisation du traitement des matériaux métalliques par laser. \\ I. Etablissement d'une méthodologie
}

\author{
J. Dietz (*) et J. Merlin \\ I.N.S.A. Lyon Bât. 502, G.E.M.P.P.M., U.A. 341 Calfetmat, F-69621, Villeurbanne Cedex, France
}

(Reçu le 7 mars 1988, révisé le 18 juillet 1988, accepté le 16 août 1988)

\begin{abstract}
Résumé. - Malgré les nombreux travaux démontrant la faisabilité des traitements thermiques par laser et les études à caractère plus théorique sur l'interaction rayonnement matériau, il est encore difficile d'exploiter scientifiquement les résultats empiriques publiés. Nous proposons donc une démarche qui devrait faciliter les analyses $a$ priori et permettre une approche plus rationnelle des traitements laser. Pour cela une caractérisation préalable du faisceau au moyen de paramètres pertinents est nécessaire. Les paramètres retenus ont été définis d'un point de vue mathématique mais sont aussi accessibles à la mesure (un minimum de 3 paramètres sont nécessaires : la puissance transportée, un rayon " équivalent » $r^{+}$, un " facteur d'étalement de la répartition d'énergie » $g(E))$. Notre démarche passe également par une modélisation de l'interaction laser-matériau à caractère analytique et tridimensionnel mais avec un formalisme simplifié très souple d'emploi. Une fois connus le coefficient d'absorption et les caractéristiques thermiques du matériau il est alors aisé d'établir des corrélations entre les paramètres de traitement (en faisceau fixe ou mobile) et les évolutions des grandeurs conditionnant la nature et l'étendue des transformations microstructurales (accroissement de température en surface, profondeur de pénétration de la chaleur, vitesse moyenne de variation de la température...). Dans les conditions habituelles de travail on peut définir 3 régimes conditionnés par les caractéristiques du matériau (diffusivité) et du faisceau $\left(r^{+}\right.$et $g(E)$ ), régimes : - transitoire (avec des lois d'évolution en $t^{1 / 2}$ ) intermédiaire (lois en $t^{1 / 4}$ ) - et stationnaire (indépendant de $t$ ). Une $2^{\mathrm{e}}$ partie traitera des possibilités qu'ouvre la méthodologie proposée.
\end{abstract}

\begin{abstract}
Despite of a great number of investigations showing the laser heat-treatments feasibility and of theoretical studies on laser beam-material interactions, a scientifical utilization of published empirical results is still difficult. Our purpose is to suggest a method which would facilitate $a$ priori analysis and allow a rational approach of laser treatments. First of all it is necessary to describe the beam with relevant parameters. Those we used mathematically are defined (based on a thermal description) and are also experimentally measurable. Three parameters are needed : the carried power, an equivalent radius $r^{+}$and a spread factor of energy distribution $g(E)$. Our approach uses an analytical tridimensional modelization of laser-material interactions with a simplified and easy to use formalism. Once the absorption coefficient and the thermal parameters of the material are known, it becomes easy to find correlations between treatment parameters (the source being mobile or stationnary) and the variations of parameters determining the nature and the extension of some microstructural transformations (surface temperature increase, heat penetration depth, average rate of temperature change..., etc.). In usual working conditions, three cases conditioned by material characteristics (thermal diffusivity) and beam characteristics $\left(r^{+}\right.$and $\left.g(E)\right)$ can be defined; there are : transient (with $t^{1 / 2}$ evolutions, $t:$ time), intermediate $\left(t^{1 / 4}\right)$ and stationnary (independent of $t$ ). A second part of this study will deal with possibilities of the approach.
\end{abstract}

\section{Introduction.}

La faisabilité de traitements thermiques en phase solide sur des alliages métalliques par apport localisé

(*) Adresse actuelle: S.I.C.N., Veurey-Voroize, F38113, France. de grandes densités d'énergie grâce à des sources lasers n'est plus à démontrer [1-4].

Reste pourtant posé le problème de la maîtrise des effets induits. Notre objectif est donc d'essayer de dégager les éléments susceptibles, d'une part de conduire à une optimisation de la mise en œuvre de 
traitements lasers (en phase solide pour l'essentiel), d'autre part de permettre une analyse métallurgique aussi rigoureuse que possible.

La condition préalable à l'atteinte d'un tel objectif est de disposer de sources lasers aux caractéristiques stables, reproductibles et connues. En effet, les évolutions spatiales et temporelles de la répartition d'énergie dans le faisceau conditionnent tous les effets induits au niveau de la zone d'interaction [5]. Pour que cette condition soit remplie, il est donc impératif de mettre au point une métrologie de faisceau adaptée aux sources de fortes puissances et susceptible de déboucher sur une caractérisation en temps réel utilisable dans le cadre de la mise en œuvre de traitements lasers. Nous consacrerons donc un paragraphe à ce problème particulier.

Lorsque l'on consulte des publications sur les traitements laser, on constate que très souvent les auteurs se contentent d'une approche empirique avec « analyse a posteriori » des résultats, la description des effets induits pouvant être assortie d'une tentative de corrélation avec certains paramètres de traitements (par exemple liaison entre le durcissement observé à $X \mathrm{~mm}$ de la surface et la puissance d'émission de la source). Ce type de démarche a pu démontrer la faisabilité d'un certain nombre de traitements mais il est maintenant nécessaire d'aller plus loin. En effet, sans un minimum de raisonnement préalable il est fort possible de dégager des corrélations n'ayant aucune valeur de généralité. Des corrélations différentes sont ainsi proposées entre la profondeur de durcissement observée sur un acier et : puissance d'émission, rayon du faisceau, vitesse de déplacement (voir par exemple [6] et [7]). En outre, il manque très souvent des informations détaillées sur les modalités de traitement (caractéristiques des sources, des échantillons, des couvertures gazeuses, etc...) ce qui rend les résultats fournis ni extrapolables ni même susceptibles d'être reproduits et aucune conclusion scientifique sérieuse ne peut alors être tirée.

Bien entendu les faiblesses d'une telle approche ont déjà conduit nombre de chercheurs à interpréter les effets induits à partir de modèles thermiques devant décrire leurs conditions expérimentales. Ces modèles consistent le plus souvent en une résolution numérique d'une équation de la chaleur avec certaines hypothèses et conditions aux limites (voir par exemple $[8,9,10])$. Cette approche facilite la prise en compte des non-linéarités de certains paramètres (en particulier les évolutions avec la température des caractéristiques du matériau ou du coefficient d'absorption du rayonnement), mais en contrepartie il devient presque toujours possible de faire correspondre numériquement un modèle avec un résultat expérimental pris isolément.

A titre d'exemple nous citerons un travail dont la méthodologie est remarquable mais où l'auteur utilise un modèle thermique avec hypothèse d'un flux de chaleur unidirectionnel, alors que les conditions expérimentales ne sont pas conformes à cette hypothèse [11]. Or la résolution d'une équation de la chaleur non linéaire par méthode d'éléments finis permet de trouver une adéquation satisfaisante entre «théorie » et expérience en introduisant une chute importante de l'absorption du rayonnement au cours du traitement, ce qui est très probablement erroné, comme l'auteur le souligne d'ailleurs.

Devant les risques d'une telle démarche, il nous paraît préférable de privilégier une approche pratiquant une analyse a priori permettant de définir des paramètres de traitement en fonction des effets induits recherchés. Cette méthodologie, déjà proposée par plusieurs auteurs $[12,13]$, devrait déboucher au niveau industriel sur une optimisation de la mise en œuvre des traitements lasers et à un niveau plus fondamental elle devrait permettre des analyses métallurgiques fines dans des conditions extrêmes encore jamais réalisées.

Pour cela il est nécessaire de définir préalablement les conditions à remplir pour atteindre les caractéristiques mécaniques ou métallurgiques désirées. Ensuite il faut établir un modèle de comportement prévisionnel de l'interaction laser-matériau (à caractère essentiellement thermique au moins dans un premier temps). Pour être efficace ce modèle devra être le plus simple possible et n'intégrer que des paramètres accessibles à la mesure en présentant toutefois une adaptabilité aussi grande que possible vis-à-vis des diverses situations pouvant se rencontrer lors de la mise en œuvre d'une catégorie donnée de traitement.

Il n'est bien entendu pas possible ici de présenter un travail exhaustif couvrant toutes les situations concevables, nous nous limiterons donc au cas simple de la realisation d'un traitement sur une couche superficielle d'un matériau métallique massif soumis à des flux d'énergie photonique de densité et de répartition variable. Pour cela nous nous appuierons sur l'abondante littérature existant dans ce domaine [14-22] en nous attachant cependant à formuler les résultats sous une forme originale qui soit la plus analytique et la plus universelle possible.

\section{Caractérisation du faisceau.}

Il est courant dans les modélisations d'admettre que les répartitions de l'énergie sont gaussiennes. Pourtant, il n'y a qu'à examiner les impacts obtenus dans des matériaux tels que le plexiglas pour se convaincre que cette description est peu réaliste pour la plupart des sources laser de puissance existant sur le marché [23]. Or, les effets induits sont conditionnés par la répartition réelle de l'énergie au niveau de la zone d'interaction au moment de l'impact avec le matériau ; une caractérisation la plus complète mais aussi 
la plus simple possible est nécessaire afin de permettre à terme un asservissement de la source. Cette répartition pouvant fortement varier sur le trajet du faisceau [24] la caractérisation devra être effectuée le plus près possible de la zone de travail avec les difficultés que cela comporte, des mesures dans une zone de focalisation risquant de perturber le faisceau.

Les répartitions d'énergie peuvent être plus ou moins complexes mais pour être utilisables en traitement thermique elles ne doivent pas présenter trop « d'accidents » qui entraîneraient obligatoirement des irrégularités dans les cycles thermiques. Nous nous sommes donc attachés à décrire des faisceaux présentant un axe de symétrie avec un maximum de densité de puissance au voisinage immédiat de cet axe (des irrégularités locales de faible amplitude inférieure à quelques \% - pouvant être négligées). Nous couvrons ainsi toute une gamme de répartitions d'énergie allant pratiquement du faisceau TEM OO (gaussienne illimitée) à des faisceaux homogènes de section rectangulaire, circulaire ou elliptique en passant par des faisceaux pouvant se décrire par des répartitions, soit gaussiennes plus ou moins diaphragmées, soit coniques circulaire ou elliptique plus ou moins tronquées ou diaphragmées... et dans une certaine mesure des répartitions de type annulaire peuvent aussi être prises en compte (voir la Fig. A1 de l'annexe 1 pour la définition des caractéristiques géométriques de l'ensemble de ces répartitions).

Il existe différents dispositifs susceptibles de caractériser un faisceau, entre autres des dispositifs à aiguille tournante donnant les variations d'énergie selon des directions particulières [25]. Il nous semble cependant que la caractérisation d'un faisceau en vue de l'introduction de paramètres dans un modèle de comportement prévisionnel ne passe peut-être par nécessairement par une analyse aussi complète et devrait pouvoir se limiter à l'évaluation d'un nombre restreint de grandeurs. Le traitement du signal issu des dispositifs précités permettrait certainement une telle évaluation mais il est possible d'accéder plus simplement aux caractéristiques essentielles du faisceau une fois précisées les grandeurs gouvernant l'interaction faisceau-matériau au voisinage immédiat de l'axe du faisceau à savoir :

- la quantité globale d'énergie transportée (qui conditionne «le volume » des effets induits)

- la densité maximale d'énergie appliquée (qui conditionne «l'intensité » des effets induits)

- l'étalement de l'énergie incidente c'est-à-dire une grandeur représentative des gradients de densité d'énergie dans la zone d'interaction (qui conditionnent les flux d'échange avec le matériau).

Cela signifie que trois mesures judicieuses doivent être susceptibles de caractériser correctement un faisceau. Ces mesures devant être aussi simples que possible nous avons retenu des évaluations de puissance transmise à travers des diaphragmes de diamètre $\phi_{i}$ ou des fentes de largeur $\varepsilon_{i}$ connus et effectuées sur le faisceau direct immédiatement en amont de la zone de focalisation.

Peuvent ainsi être évaluées [26] :

- la puissance totale incidente $P$ (correspondant à un diaphragme $\phi_{\infty}$ ),

- la puissance au centre du faisceau $P_{1}$, transmise à travers un diaphragme de diamètre le plus petit possible ( $\phi_{1}=0,5$ à $1 \mathrm{~mm}$ en pratique),

- la puissance $P^{+}$transmise à travers un diaphragme de diamètre $\phi^{+}=\phi_{1} \sqrt{P / P_{1}}$,

- les puissances $P_{\varepsilon}(j)$ transmises à travers une fente de largeur $\varepsilon$ orientée selon plusieurs directions dans le plan d'interaction.

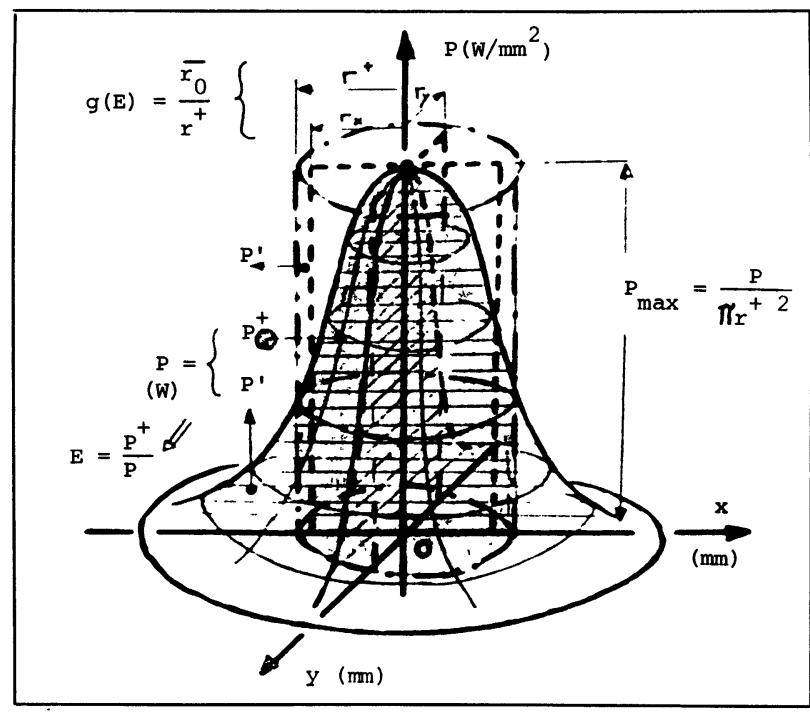

Fig. 1. - Représentation des paramètres caractéristiques de la répartition de la puissance dans un faisceau laser.

[Representation of specific parameters of power repartition in a laser beam.]

De ces mesures peuvent être déduits un certain nombre de paramètres représentatifs de la répartition d'énergie dans le plan d'observation (Fig. 1) :

- la densité de puissance maximale $p_{M}=$ $4 P_{1} / \pi \phi_{1}^{2}$,

- deux paramètres représentatifs des dimensions du faisceau :

- un rayon équivalent $r^{+}$, correspondant au rayon du faisceau circulaire homogène transportant la même énergie et ayant $p_{M}$ identique ; on aura $r^{+}=\left(\phi_{1} / 2\right) \sqrt{P / P_{1}}$;

- une distance moyenne $\bar{r}_{\mathrm{o}}$ représentant la moyenne des distances équivalentes de la répartition d'énergie dans $n$ directions $O x$ autour de l'axe du faisceau :

$$
\bar{r}_{\mathrm{o}}=\frac{1}{2 n \varepsilon p_{\mathrm{M}}} \sum_{j=1}^{n} P_{\varepsilon}(j)=\frac{\pi \phi_{1}^{2}}{8 n \varepsilon P_{1}} \sum_{j=1}^{n} P_{\varepsilon}(j)
$$


- deux paramètres représentatifs de la forme de la répartition d'énergie, toujours à l'aplomb de l'axe du faisceau :

- un « étalement » moyen : $E=P^{+} / P$

- un facteur d'étalement central :

$$
g(E)=\bar{r}_{\mathrm{o}} / r^{+}=\frac{\pi \phi_{1}}{4 n \varepsilon \sqrt{P \cdot P_{1}}} \sum_{j=1}^{n} P_{\varepsilon}(j) .
$$

Pour différentes configurations les évaluations théoriques et les significations physiques de ces paramètres sont présentées dans les annexes 1 et 2 . Les principaux résultats sont rassemblés dans le tableau I.

Pour une répartition de type annulaire, les grandeurs définies précédemment peuvent être généralisées, $p_{\mathrm{M}}$ correspondant à la densité de puissance maximale sur l'anneau, $E$ et $g(E)$ restant définis par rapport à l'axe du faisceau (voir annexe 1).

Expérimentalement les évaluations des $P_{\varepsilon}(j)$ et par conséquent de $\bar{r}_{\mathrm{o}}$ et $g(E)$ étant relativement délicates, on pourra éventuellement se limiter à une détermination de $E$ et à admettre la conjecture selon laquelle existent des relations biunivoques entre $g(E)$ et $E$ dépendant seulement du fait que les répartitions présentent un minimum ou un maximum central ; ce point est repris plus en détails dans l'annexe 3. Par contre il est beaucoup plus délicat d'introduire $E$ dans les modèles de comportement prévisionnel. Dans la suite, quelles que soient les distributions d'énergie, nous décrirons les faisceaux en un plan particulier $w$ sur leur trajet au moyen des trois paramètres suivants issus de la mesure : puis- sance totale $P$, rayon équivalent $r_{w}^{+}$, facteur d'étalement $g(E)$. Ces évaluations ne pouvant être effec-; tuées qu'en dehors d'une zone de focalisation, si l'on travaille dans une telle zone il faudra bien entendu en tenir compte. En fait, si l'on néglige les effets d'interférence et d'aberrations seul $r_{w}^{+}$sera modifié $\left(r^{+}\right.$utile $=\mu r_{w}^{+}$, le coefficient de proportionnalité $\mu$ étant susceptible d'être évalué expérimentalement [26] à partir de considérations simples d'optiques géométrique et gaussienne [24]). Dans la suite seules interviendront les modifications du rayon utile $r^{+}$, pouvant résulter soit de celles de $r_{w}^{+}$(en agissant sur l'émission de la source), soit de celles de $\mu$ (en jouant sur l'optique de focalisation).

\section{Caractérisation microstructurale des effets induits par un traitement laser superficiel.}

Tout comme pour la caractérisation du faisceau il faut commencer par dégager les grandeurs nécessaires et suffisantes pour définir le traitement thermique devant être mis en œuvre. Comme nous l'avons déjà écrit, nous nous intéresserons ici essentiellement à des traitements à l'état solide, à caractère localisé et superficiel sur des matériaux métalliques massifs sur lesquels nous chercherons à réaliser une transformation microstructurale donnée $\mathcal{M}$, sur une profondeur $e$, et une étendue latérale $\Phi$ (voir schéma Fig. 2a).

D'un point de vue métallurgique la transformation est conditionnée par le cycle thermique subi par le matériau. Les paramètres essentiels pour un traitement s'étendant sur une durée $t_{\mathrm{T}}$ sont : la température maximale $T_{0}$ atteinte durant ce laps de temps,

Tableau I. - Paramètres caractéristiques de quelques répartitions d'énergie (voir l'annexe 1 pour la définition des répartitions).

[Characteristical parameters of several energy repartitions (see annex 1 for definitions of the repartitions.]

\begin{tabular}{|l|c|c|c|c|}
\hline Répartition & $E$ & $g(E)$ & $r^{+}$ & $\bar{r}_{\mathrm{o}}$ \\
\hline Gaussienne infinie $\left(G_{\infty}\right)$ & $1-1 / e \simeq 0,63$ & $\sqrt{\pi} / 2 \simeq 0,886$ & $r_{(1 / e)}$ & $(\sqrt{\pi} / 2) r_{(1 / e)}$ \\
\hline Gaussienne diaphragmée $G(1 / e)$ & 0,74 & 0,94 & $0,8 r_{(1 / e)}$ & $0,75 r_{(1 / e)}$ \\
\hline Homogène circulaire (HC) & 1 & 1 & $R$ & $R$ \\
\hline Homogène carrée (HK) & 0,91 & 0,995 & $\begin{array}{c}(2 / \sqrt{\pi}) a \\
\simeq 1,13 a\end{array}$ & $\begin{array}{c}(4 / \pi) \operatorname{Ln}(\operatorname{tg}(3 \pi / 8)) a \\
\simeq 1,12 a\end{array}$ \\
\hline Conique circulaire (CC) & 0,615 & $\sqrt{3} / 2 \simeq 0,866$ & $(\sqrt{3} / 3) R$ & $R / 2$ \\
\hline Homogène rectangulaire HR(4) $\beta^{\prime}=4$ & 0,55 & 0,87 & $(4 / \sqrt{\pi}) a$ & $1,96 a$ \\
\hline Homogène elliptique $\mathrm{HE}(4) \beta^{\prime}=4$ & 0,59 & 0,89 & $2 a$ & $1,78 a$ \\
\hline Conique elliptique $\mathrm{CE}(3) \beta^{\prime}=3$ & 0,53 & 0,80 & $a$ & $0,80 a$ \\
\hline Tronconique circul. TC(0,5) $\beta^{\prime}=0,5$ & 0,84 & 0,98 & $0,76 R$ & $(3 / 4) R$ \\
\hline
\end{tabular}



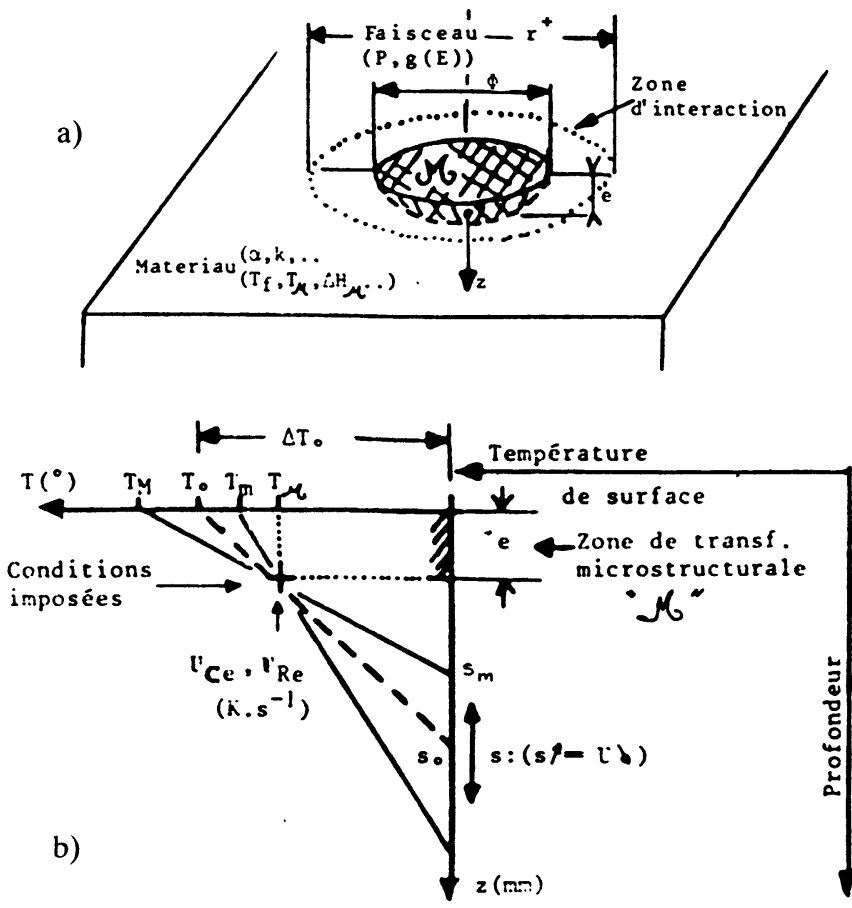

Fig. 2. - a) Vue générale de la zone d'interaction faisceau-matériau et de la zone traitée $(\mathcal{M})$. b) Représentation schématique des profils thermiques conduisant à une épaisseur transformée $e$.

[a) Global view of the area of beam-material interaction and of the treated area ( $\mathcal{N})$. b) Schematical representation of thermal profiles producing a transformed thickness $e$.]

les vitesses moyennes de chauffage et de refroidissement, $U_{\mathrm{C}}$ et $U_{\mathrm{R}}$ et le temps $t_{\mathrm{I}}$ de maintien « isotherme » à la température $T_{0}$. Selon la nature de la transformation recherchée, tel ou tel de ces paramètres pourra jouer un rôle prépondérant.

Il faut aussi tenir compte des spécificités du traitement laser des métaux en phase solide. En particulier l'énergie photonique peut être considérée comme convertie en énergie thermique dans une couche superficielle n'excédant pas quelques dizaines de nanomètres [5] puis cette énergie thermique s'écoule dans le matériau par diffusion. Le cycle thermique suivi sera donc différent en tout point du solide; il . est toutefois évident que dans le cas d'interaction avec un faisceau présentant un maximum de densité d'énergie au voisinage de son axe, plus on s'éloignera du centre de la zone d'interaction (directions $O x$ ) et plus la température atteinte sera faible ; de même cette température diminuera lorsque l'on pénétrera vers l'intérieur du matériau (direction $\mathrm{O} z$ ).

Par suite, vouloir une transformation $\mathcal{M}$ sur une étendue donnée (distance $\Phi / 2$ ou $e$ respectivement dans les directions $\mathrm{O} x$ ou $\mathrm{O} z$ ) impose l'atteinte en ces lieux particuliers d'une température minimale $T_{\mathrm{m}}$ susceptible d'induire la transformation (par exemple la température eutectoïde pour un acier). La température maximale $T_{0}$ observée en surface devra être supérieure à $T_{\mathrm{m}}$ sans dépasser une température limite $T_{\mathrm{M}}$ (atteinte de la fusion par exemple). La réalisation d'un tel traitement passe par l'obtention d'un certain profil de température pouvant être défini par l'accroissement de température observé en surface : $\Delta T_{\mathrm{S}}=T_{0}-T_{\text {ambiante }}$ et par un gradient moyen de la température, ou encore par une distance de propagation de la chaleur $s$ dans la direction considérée (voir Fig. 2b). Cette distance étant obligatoirement reliée aux possibilités de diffusion thermique du matériau, donc au temps de traitement, à un profil spatial de température correspondront des vitesses de variation de température $U_{\mathrm{C}}$ au chauffage et $U_{\mathrm{R}}$ au refroidissement. Si ces grandeurs jouent un rôle primordial dans la transformation $\mathcal{N}$, tous ces profils ne seront pas équivalents du point de vue métallurgique. En particulier à une vitesse minimale de refroidissement $U_{\mathrm{Rm}}$ imposée correspondront un temps de traitement maximal et par voie de conséquence une profondeur $s_{\mathrm{R}}$ maximale ce qui restreindra d'autant le choix des profils possibles et pourra même aboutir à l'impossibilité de réalisation de $\mathcal{K}$ sur l'étendue envisagée initialement si $s_{\mathrm{R}}$ est inférieure à la distance de propagation minimale $s_{\mathrm{m}}$ imposée par $T_{\mathrm{M}}$ et $e$.

De toute façon la plupart des effets induits à l'aplomb du faisceau doivent pouvoir être définis sans ambiguité à partir de :

- l'accroissement maximal de températưre $\Delta T_{\mathrm{S}}$ à atteindre,

- la distance de propagation $s$ nécessaire et suffisante dans la direction $\mathrm{O} z$,

- et éventuellement l'ordre de grandeur $\mathbf{U}$ des vitesses de variation en température $U_{\mathrm{C}}$ et $U_{\mathrm{R}}$. Les modèles thermiques que nous allons maintenant chercher à établir devront donc permettre d'accéder à ces grandeurs en fonction des différents paramètres nécessaires à la mise en œuvre du traitement.

\section{Les modèles thermiques permettant l'optimisation des traitements lasers en phase solide de matériaux métalliques massifs.}

4.1 Présentation du PROBlÈMe. - De tels modèles devront en principe décrire le comportement thermique à chaque instant et en tout point du solide en n'oubliant toutefois pas que c'est avant tout le caractère prévisionnel qui est recherché. Pour être le plus simple possible ils devront être adaptés au problème particulier auquel on est confronté c'est-àdire dans le cas présent à la réponse thermique du solide semi-infini soumis localement à un flux énergétique superficiel $P$. Cela implique que les dimensions du solide à traiter soient grandes vis-à-vis des dimensions du faisceau incident ou encore qu'un couplage thermique soit réalisé avec un support 
conducteur de dimensions suffisantes pour que l'énergie incidente puisse s'y dissiper (il est nécessaire de garder présent à l'esprit que les distances maximales de diffusion de la chaleur durant un temps $t_{\mathrm{T}}$ dans un matériau de diffusivité thermique $\alpha$ sont toujours de l'ordre de $2 \sqrt{\alpha t_{\mathrm{T}}}$ [15], ce qui permet de définir les conditions géométriques à remplir).

Ces modèles devant être capables de relier les évolutions temporelles de $\Delta T_{\mathrm{S}}, s, U_{\mathrm{C}}$ et $U_{\mathrm{R}}$ aux différents paramètres gouvernant le traitement, il faut établir la liste de ceux-ci en essayant de la restreindre le plus possible et de la limiter à des grandeurs accessibles à la mesure (en temps réel si possible) ou sur lesquelles il sera possible d'agir durant la réalisation du traitement.

Nous avons ainsi retenu :

- pour le faisceau (outre ses caractéristiques intrinsèques, telle que la longueur d'ondes du rayonnement $\lambda$, qui n'interviendra pas explicitement dans les modèles) les grandeurs issues de la caractérisation :

- $P$ puissance totale transportée,

- $r^{+}$rayon équivalent (dans la zone de travail),

- $g(E)$ facteur d'étalement central (également au voisinage de la zone de travail);

- pour le matériau (outre sa nature et ses caractéristiques géométriques) :

- $k$ conductivité thermique,

- $\alpha$ diffusivité thermique ;

- pour l'interaction rayonnement-matériau :

- $\rho$ rendement énergétique global (rapport entre l'énergie absorbée par le matériau et celle émise par la source, qui prend en compte d'éventuelles pertes dues à la chaîne de transport)

- $\tau$ temps d'interaction entre le faisceau et le matériau défini comme correspondant soit à la durée d'une impulsion ou de l'éclairement dans le cas d'un faisceau pulsé ou continu et fixe par rapport au matériau à traiter, soit au temps d'éclairement équivalent $\tau^{+}=2 r_{x} / v$ dans le cas d'un faisceau continu en déplacement relatif selon l'axe $x$, à la vitesse $v$ par rapport au matériau (avec $r_{x}$ paramètre représentatif de la dimension du faisceau dans la direction de déplacement relatif - voir annexe 1).

Ces sept paramètres sont essentiels pour la mise en œuvre du traitement mais les possibilités d'action sur ceux-ci sont très diverses :

- $\alpha$ et $k$ sont imposés par la nature du matériau, de plus ils ne sont pas toujours très bien connus et peuvent évoluer avec la température ; toutefois afin de ne pas compliquer les modèles et conformément aux objectifs fixés nous admettrons ces coefficients constants durant la durée du traitement, une valeur moyenne devant permettre de définir le comportement avec une précision acceptable. Cette hypothèse est en particulier confortée par les études de Kou [27] qui a montré que la prise en compte de la nonlinéarité de ces paramètres ne modifiait pas notablement les prévisions de comportement.

- le rendement $\rho$ pas toujours très bien connu, fonction de la nature des revêtements déposés en surface du matériau et lui aussi variable au cours du traitement, sera admis comme constant. Pratiquement il y a intérêt à ce que ce coefficient soit aussi élevé que possible en améliorant les dispositifs optiques et les dépôts absorbants. Dans ce domaine aussi un important effort de caractérisation est à accomplir (nécessité d'approfondir des études du type de celles entreprises dans différents laboratoires $[28,29])$;

- la loi de distribution de l'énergie dans la zone d'interaction est traduite par $g(E)$ et dans l'état actuel des techniques il est délicat de modifier volontairement ce paramètre en cours de traitement, il faut par contre conserver présent à l'esprit que cette distribution spatiale est souvent fonction du régime de puissance d'émission de la source et risque donc de ne pas être totalement indépendante de $P$.

Les seuls paramètres pouvant être modifiés relativement simplement pour la réalisation d'un traitement sont donc $P, r^{+}$et $\tau$ mais là encore ils ne sont pas obligatoirement indépendants : $r^{+}$peut évoluer avec $P$ comme $g(E)$. Nous avons pu montrer de telles évolutions dans le cas d'une source $\mathrm{CO}_{2}$ continue CILAS CI 4000 , pour $P$ variant de $300 \mathrm{~W}$ à $3,5 \mathrm{~kW}$ [26]. Enfin dans le cas d'un faisceau en mouvement relatif par rapport au matériau $r^{+}$et $\tau$ sont obligatoirement interdépendants par l'intermédiaire de $v$.

Du point de vue de l'utilisateur deux situations sont plus spécialement intéressantes à analyser :

- soit la détermination de l'ensemble des paramètres de traitement qui pour une source et un matériau donnés sont susceptibles d'induire un accroissement maximal $\Delta T_{\mathrm{S}}$ de température en surface avec les valeurs correspondantes des autres grandeurs conditionnant la transformation microstructurale : $s, U_{\mathrm{C}}$ et $U_{\mathrm{R}}$;

- soit toujours pour une source et un matériau donnés, la détermination des trajectoires d'évolution à l'aplomb du faisceau de $\Delta T_{\mathrm{S}}$, $s$, et $U_{\mathrm{C}}$ au cours du temps durant l'application d'un flux énergétique $P_{i}$ donné et pour des valeurs discrètes des autres paramètres.

Ces deux situations correspondent en fait à deux approches d'un même problème pouvant être traité par un seul modèle à partir du moment où est considéré le rapport :

$$
\Delta T_{\mathrm{S}} / P=f\left(t_{\mathrm{i}}\right)_{r^{+}, g(E), \rho, \alpha, k}
$$

avec $t_{\mathrm{i}}$ temps d'observation ou d'éclairement selon la situation envisagée. 
De même on s'intéressera à $s=f^{\prime}\left(t_{\mathrm{i}}\right)_{r^{+}}, \ldots$ en remarquant que $s$ peut être relié au gradient moyen de température $\gamma$ dans la direction considérée et que par application de la loi de Fourier $s$ est proportionnelle à $\left(\pi r^{+2} k / \rho\right)\left(\Delta T_{\mathrm{S}} / P\right)$. Durant l'application d'un flux énergétique constant le coefficient de proportionnalité vaut $\pi / 2$ (d'après [15]), par conséquent $s=\left(\pi^{2} r^{{ }^{2}} k / 2 \rho\right)\left(\Delta T_{\mathrm{S}} / P\right)$.

En surface, les vitesses $U_{\mathrm{C}}$ et $U_{\mathrm{R}}$ sont constamment variables au cours d'un cycle thermique. $U_{\mathrm{C}}$ débute avec une valeur maximale égale à $\rho p_{M} \alpha / k i \simeq$ $10^{10} \mathrm{~K} . \mathrm{s}^{-1}$, régime dit «calorimétrique » tant que $s$ est inférieure à la profondeur $i$ de pénétration du rayonnement [30] et décrô̂t continuement jusqu'au moment de l'atteinte de la température maximale. $U_{\mathrm{R}}$ varie d'une manière similaire lors du refroidissement. Nous nous contenterons donc d'apprécier un comportement moyen $\tilde{U}$ de $U_{\mathrm{C}}$ et $U_{\mathrm{R}}$ en admettant $\tilde{U}=\Delta T_{\mathrm{S}} / \tilde{t}$ avec $\tilde{t}$ temps sur lequel l'essentiel de la variation de température s'est effectué.

Remarquons qu'au voisinage de $\Delta T_{\mathrm{S}}$ cette définition tend à majorer $U_{\mathrm{C}},\left(U_{\mathrm{C}}=\tilde{U} / a\right)$ et à minorer $U_{\mathrm{R}},\left(U_{\mathrm{R}}=a \tilde{U}\right)$. Le coefficient « $a »$, fonction du matériau et des paramètres de traitement, reste de l'ordre de 2 en général. Enfin, à une profondeur $e$, inférieure à $s / 2$, la vitesse $U_{e}$ pourra être approximée par $\tilde{U}\left(\Delta T_{e} / \Delta T_{\mathrm{S}}\right)$, avec $\Delta T_{e}$ accroissement de température observé à la profondeur $e$.

\subsection{DESCRIPTION DES DIFFÉRENTS RÉGIMES THER-} MIQUES. - La résolution de l'équation de la chaleur avec les hypothèses et les conditions aux limites correspondant au type de traitement envisagé permet de déterminer les différentes relations (voir annexe 2). Nous avons vérifié la conformité de nos résultats avec certains de ceux déjà publiés dans ce domaine mais surtout, nous avons cherché à les présenter sous une forme originale synthétisant l'ensemble des comportements au moyen d'expressions à caractère analytique permettant de prévoir des valeurs à mieux que $10 \%$ et cela quelle que soit la loi de distribution de l'énergie au sein du faisceau (dans les limites précédemment définies, bien entendu).

Pour cela il est nécessaire d'introduire le paramètre $t_{\mathrm{c}}$, «temps critique ", défini à partir de l'intersection des limites asymptotiques des comportements thermiques du matériau considéré comme un solide semi-infini pour $t \rightarrow 0$ et $t \rightarrow \infty$ (voir annexe 2); ce paramètre est décrit par la relation :

$$
t_{\mathrm{c}}=\left[\pi r^{+^{2}} / 4 \alpha\right] g(E)^{2}=\pi \bar{r}_{\mathrm{o}}^{2} / 4 \alpha .
$$

Selon la valeur relative du temps $t_{\mathrm{i}}$ (d'observation ou d'éclairement) vis-à-vis de $t_{\mathrm{c}}$ trois régimes décrits par des lois distinctes seront à considérer (voir annexe 2). L'évaluation du $t_{\mathrm{c}}$ nécessaire ou imposé pour un traitement donné constitue donc un préalable à toute étude. Nous devons également remarquer que $t_{c}$, très sensible aux variations de dimensions du faisceau, ne dépendra finalement qu'assez peu de la forme de la répartition d'énergie $\left(t_{\mathrm{c}}\right.$ augmente d'environ $25 \%$ en passant d'une répartition gaussienne illimitée à une répartition homogène circulaire ayant même rayon équivalent).

a) $\mathrm{Si} t_{\mathrm{i}} \gg t_{\mathrm{c}}$, nous désignerons ce régime comme étant quasi stationnaire ou encore «permanent » :

$$
\Delta T_{\mathrm{S}} / P=\rho g(E) / \pi k r^{+}=\rho g(E)^{2} / \pi k \bar{r}_{\mathrm{o}}
$$

soit encore $\quad \Delta T_{\mathrm{S}} / \Delta T_{\mathrm{L}}=P_{\mathrm{L}} / P=1$

avec

$$
\begin{aligned}
\Delta T_{\mathrm{L}} & =\left[\rho g(E) / \pi k r^{+}\right] P_{\mathrm{i}} \\
& =\left[\rho g(E)^{2} / \pi k \bar{r}_{\mathrm{o}}\right] P_{\mathrm{i}}
\end{aligned}
$$

température limite atteinte après application d'une puissance donnée $P_{\mathrm{i}}$ et

$$
\begin{aligned}
P_{\mathrm{L}} & =\left[\pi k r^{+} / \rho g(E)\right] \Delta T_{\mathrm{i}} \\
& =\left[\pi k \bar{r}_{\mathrm{o}} / \rho g(E)^{2}\right] \Delta T_{\mathrm{i}}
\end{aligned}
$$

puissance minimale à communiquer pour atteindre un accroissement de température de surface $\Delta T_{\mathrm{i}}$ donné.

Ce régime est donc caractérisé par une indépendance de $\Delta T$ vis-à-vis du temps; par voie de conséquence il en est de même pour $s$ qui se stabilise à une valeur limite $s_{\mathrm{L}}$ :

$$
s_{\mathrm{L}}=\pi g(E) r^{+} / 2=\pi \bar{r}_{0} / 2 .
$$

Pratiquement on a : $r^{+}<s_{\mathrm{L}}<1,55 r^{+}$, et cela indépendamment de la nature du matériau.

En ce qui concerne $\tilde{U}$, lorsque $t \rightarrow \infty, \tilde{U} \rightarrow 0$, cependant l'essentiel des variations en température s'effectue durant un temps de l'ordre de $t_{\mathrm{c}}$, d'où la définition d'une vitesse limite apparente $\tilde{U}_{\mathrm{L}}=$ $\Delta T_{\mathrm{L}} / t_{\mathrm{c}}$ soit :

$$
\begin{aligned}
\tilde{U}_{\mathrm{L}} & =4 \alpha \rho P_{\mathrm{i}} / \pi^{2} g(E) k r^{+^{3}} \\
& =4 \alpha \rho g(E)^{2} P_{\mathrm{i}} / \pi^{2} k \bar{r}_{\mathrm{o}}^{3} .
\end{aligned}
$$

Ce régime correspond à l'obtention d'un équilibre entre le flux énergétique incident et les possibilités de dissipation dans le matériau. Les dimensions du faisceau et le gradient d'énergie au sein de ce faisceau jouent un rôle capital vis-à-vis de ce régime. Ainsi pour une puissance transportée donnée, plus l'énergie sera concentrée sur une petite surface et plus la température maximale atteinte pourra être élevée ; mais pour un même rayon équivalent cette température sera d'autant plus élevée que l'étalement sera grand. Ainsi un faisceau gaussien provo- 
a)
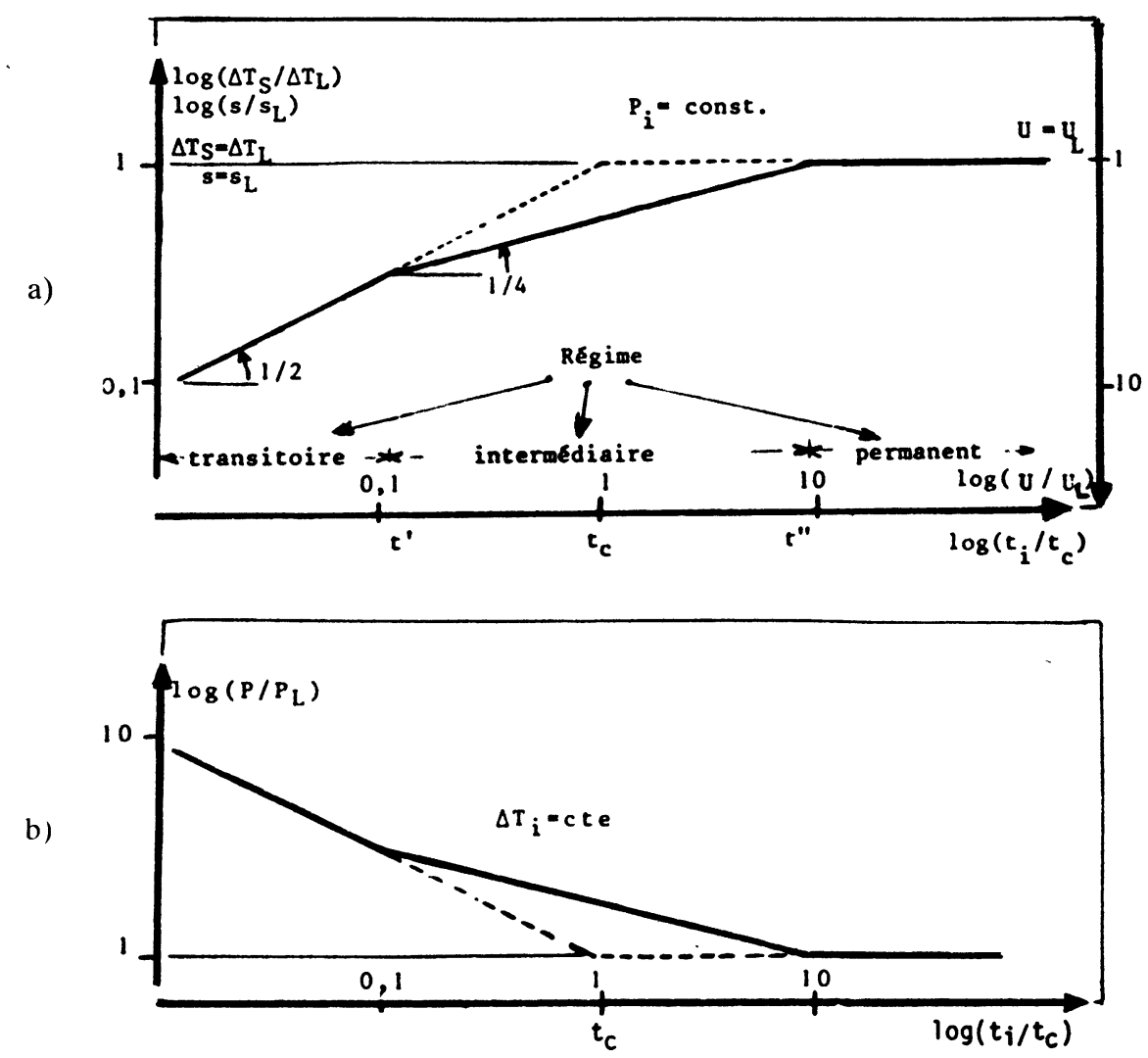

Fig. 3. - a) Diagramme universel « isoflux » représentant $\Delta T_{\mathrm{s}}, s$ et $U$ pour toute répartition à maximum central, après application d'un flux $P_{i}=$ Cte. b) Diagramme "pseudo-isotherme » représentant la puissance $P$ à fournir pour atteindre une température $\Delta T_{\mathrm{i}}$, en fonction du temps d'éclairement.

[Universal « isoflux » diagram showing $\Delta T_{\mathrm{S}}, s$ and $U$ for any repartition with a central maximum, after application of a flux $P_{i}=$ Cte. b) «Pseudo-isothermal » diagram showing the power $P$ to be supplied to reach a temperature $\Delta T_{\mathrm{i}}$, as a function of lighting time.]

quera un accroissement de température environ $10 \%$ inférieur à celui obtenu avec un faisceau homogène circulaire équivalent. Un comportement similaire s'observera pour la profondeur limite de pénétration de la chaleur.

b) Si $t_{\mathrm{i}} \ll t_{\mathrm{c}}$, nous désignerons ce régime comme étant «transitoire »; il est caractérisé par :

$$
\begin{gathered}
\Delta T_{\mathrm{S}} / P=2 \rho \sqrt{\alpha t_{\mathrm{i}}} / \pi^{1,5} k r^{+^{2}} \\
=2 \rho g(E)^{2} \sqrt{\alpha t_{\mathrm{i}}} / \pi^{1,5} k \bar{r}_{\mathrm{o}}^{2} \\
s=\sqrt{\pi \alpha t_{\mathrm{i}}} \\
\tilde{U}=\Delta T_{\mathrm{S}} / t_{\mathrm{i}}=\left[2 \rho P / \pi^{1,5} k r^{+2}\right] \sqrt{\alpha / t_{\mathrm{i}}} \\
=\left[2 \rho g(E)^{2} P / \pi^{1,5} k \bar{r}_{\mathrm{o}}^{2}\right] \sqrt{\alpha / t_{\mathrm{i}}}
\end{gathered}
$$

soit encore

$$
\begin{aligned}
\Delta T_{\mathrm{S}} / \Delta T_{\mathrm{L}} & =P_{\mathrm{L}} / P=s / s_{\mathrm{L}} \\
& =\tilde{U}_{\mathrm{L}} / \tilde{U}=\left(t_{\mathrm{i}} / t_{\mathrm{c}}\right)^{1 / 2} .
\end{aligned}
$$

Ce régime correspond à la diffusion thermique unidirectionnelle dans un solide semi-infini soumis à une densité de puissance $p=p_{M}=\rho P / \pi r^{+^{2}}$ uni- forme sur toute sa surface. Quelle que soit la distribution du flux d'énergie le comportement en un point de la surface du matériau traité n'est donc alors fonction que de la densité de puissance localement communiquée : $\Delta T_{\mathrm{S}} / p_{\mathrm{M}}=2 \sqrt{\alpha t_{\mathrm{i}}} / k$. Noter également que dans ce régime, le gradient moyen de température est constant $\left(\Delta T_{\mathrm{S}} / s=2 p_{\mathrm{M}} / \pi^{1.5} k\right)$. La vitesse moyenne de variation de température sera maximale pour $\tilde{U}_{\mathrm{M}}=\Delta T_{\mathrm{i}} / t_{\mathrm{m}}$, où $t_{\mathrm{m}}$ représente le temps minimal d'éclairement susceptible de conduire à $\Delta T_{\mathrm{i}}$ avec la puissance maximale disponible $P_{\text {Max }}$.

c) Si $t_{\mathrm{i}} \simeq t_{\mathrm{c}}$, plus précisément pour $t_{\mathrm{i}}$ tel que : $t^{\prime} \leqslant t_{\mathrm{i}} \leqslant t^{\prime \prime}$, le régime sera qualifié d'intermédiaire en prenant $t^{\prime}=\left[g(E)^{8} / w\right] t_{\mathrm{c}}$ et $t^{\prime \prime}=\left[w / g(E)^{8}\right] t_{\mathrm{c}}$ avec $w=4$ en faisceau fixe et $w=6$ en faisceau mobile (voir annexe 2). Pratiquement, $t^{\prime}$ reste de l'ordre de 0,025 à $0,25 t_{c}$ et $t^{\prime \prime}$ de l'ordre de 4 à $40 t_{\mathrm{c}}$.

Le comportement pourra alors être approximé à environ $10 \%$ en prenant

$$
\begin{aligned}
\Delta T_{\mathrm{S}} / \Delta T_{\mathrm{L}} & =P_{\mathrm{L}} / P=s / s_{\mathrm{L}}=\tilde{U}_{\mathrm{L}} / \tilde{U} \\
& =\left[g(E)^{2} / w^{1 / 4}\right]\left(t_{\mathrm{i}} / t_{\mathrm{c}}\right)^{1 / 4}
\end{aligned}
$$


Noter que ce régime sera d'autant plus étendu que le facteur d'étalement sera petit ou encore que l'on travaillera en faisceau mobile.

4.3 SYNTHĖSE DU FORMALISME PROPOSÉ. - Les différents régimes peuvent être rassemblés sur un même graphique où sont portés $\Delta T_{\mathrm{S}} / \Delta T_{\mathrm{L}}$ (ou $\left.s / s_{\mathrm{L}}\right)=F\left(t_{\mathrm{i}} / t_{\mathrm{c}}\right)$ et $\tilde{U} / \tilde{U}_{\mathrm{L}}=\left[F\left(t_{\mathrm{i}} / t_{\mathrm{c}}\right)\right]^{-1}$, condition « isoflux » (Fig. 3a), ainsi que $P / P_{\mathrm{L}}=\left[F\left(t_{\mathrm{i}} / t_{\mathrm{c}}\right)\right]^{-1}$, condition «pseudo-isotherme» (Fig. 3b), avec :

$$
\begin{array}{r}
F\left(t_{\mathrm{i}} / t_{\mathrm{c}}\right)=1 \text { pour } t_{\mathrm{i}} / t_{\mathrm{c}} \geqslant w / g(E)^{8} \\
F\left(t_{\mathrm{i}} / t_{\mathrm{c}}\right)=\left[g(E)^{2} / w^{1 / 4}\right]\left(t_{\mathrm{i}} / t_{\mathrm{c}}\right)^{1 / 4} \\
\operatorname{pour} g(E)^{8} / w \leqslant t_{\mathrm{i}} / t_{\mathrm{c}} \leqslant w / g(E)^{8} \\
F\left(t_{\mathrm{i}} / t_{\mathrm{c}}\right)=\left(t_{\mathrm{i}} / t_{\mathrm{c}}\right)^{1 / 2} \text { pour } t_{\mathrm{i}} / t_{\mathrm{c}} \geqslant g(E)^{8} / w .
\end{array}
$$

Il devient ainsi possible de comparer immédiatement les réponses d'un matériau à des flux énergétiques pour lesquels $P$ et $r^{+}$sont identiques mais avec des répartitions d'énergie différentes. Sur la figure 4a sont ainsi comparés au faisceau homogène cylindrique $(g(E)=1)$, un faisceau gaussien infini $(g(E)=\sqrt{\pi} / 2)$ et un faisceau pour lequel $g(E)=$ 0,75 . En régime transitoire, les comportements sont identiques mais $t_{\mathrm{c}}$ décroît avec $g(E)^{2}$; l'étendue du régime intermédiaire se trouve amplifiée et au niveau du régime permanent, "l'efficacité » est proportionnelle à $g(E)$. On doit cependant remarquer que notre approche qui fournit une indication sur les accroissements maximaux de température observables en surface, présente quand même une certaine ambiguïté vis-à-vis de la localisation de cet accroissement maximal. En effet, si pour les faisceaux à maximum central il n'y a pas de problèmes, par contre pour des faisceaux de type annulaire, ce niveau s'observera à l'aplomb du maximum de l'anneau en régime transitoire et tendra à se situer dans l'axe du faisceau en régime stationnaire. Par ailleurs les relations proposées sont également utilisables que le faisceau soit fixe ou mobile (voir annexe 2), mais en faisceau mobile ce diagramme ne permettra pas la détermination de l'évolution temporelle de la température en un point particulier de la surface; seule la température maximale atteinte pendant le passage du faisceau pourra être évaluée. Ces limitations nous paraissent cependant assez formelles en ce qui concerne la plupart des applications envisagées en traitement des matériaux pour lesquelles c'est plus l'amplitude de l'effet qui compte

a)

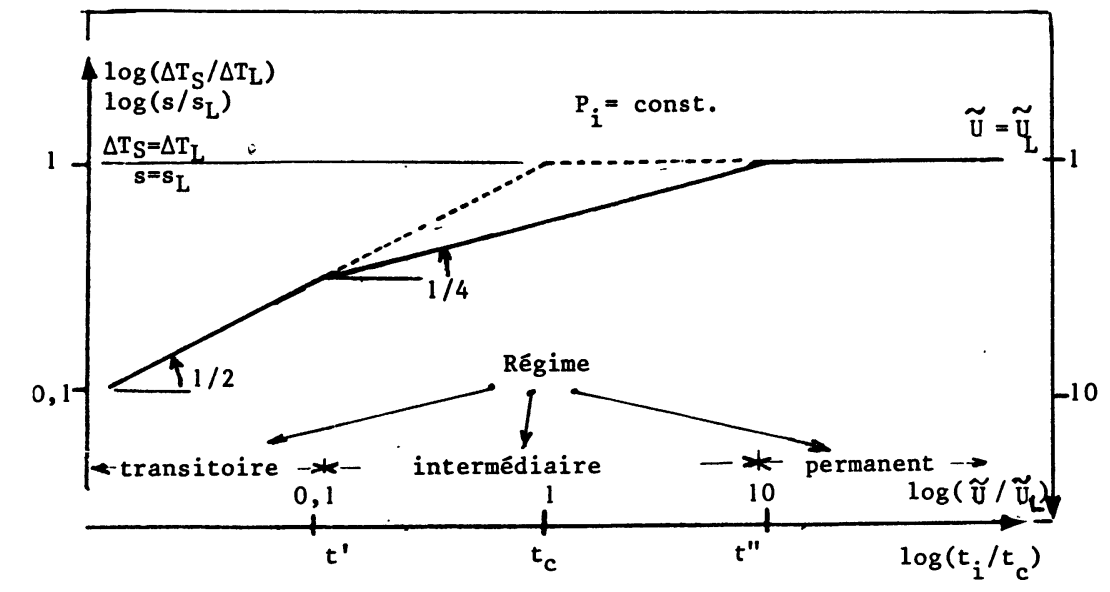

b)

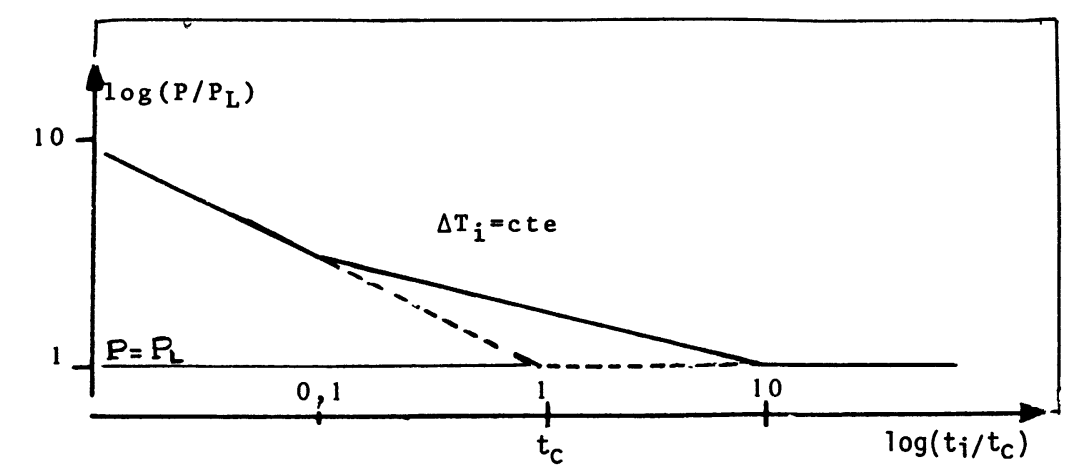

Fig. 4. - a) Comparaison des diagrammes «isoflux» pour 3 répartitions différentes. b) Evolution du diagramme « isoflux » pour une répartition donnée fixe ou mobile en fonction du temps d'interaction.

[a) Comparison of 3 «isoflux » diagrams for 3 different repartitions. b) Evolution of the « isoflux » diagram for a given repartition, moving or stationnary, with the interaction time.] 
que sa localisation précise. Remarquer également que lorsque le temps d'interaction est de l'ordre de $t_{c}$, toutes choses étant égales par ailleurs, la température maximale atteinte en faisceau mobile sera environ $10 \%$ inférieure à celle susceptible d'être atteinte en faisceau fixe (voir Fig. 4b).

Notre approche permet également d'aborder des situations plus complexes et plus proches de la réalité que celle du simple solide semi-infini soumis à un flux superficiel constant d'énergie et à une propagation diffusionnelle de l'énergie en son sein.

Ainsi une transformation structurale $(\delta \rightarrow \beta)$ peut être prise en compte dans le formalisme proposé à partir du moment où sa chaleur de transformation $\left(\Delta H_{\delta \beta}\right)$ est connue. Il sera en effet toujours possible de définir un accroissement de température équivalent à la transformation $\left(\Delta T^{\prime}=\Delta H_{\delta \beta} \cdot k / \alpha\right)$ [21]. Ainsi pour le passage à l'état liquide, $\Delta T^{\prime} \simeq$ $0,4 T_{\mathrm{F}}(\mathrm{K})$ comme nous l'avons proposé [30]; $\Delta T^{\prime}$ peut être positif ou négatif selon la nature de la transformation. Dans les différentes relations proposées $\Delta T$ doit alors être remplacé par l'accroissement de température équivalent résultant de l'interaction rayonnement-matériau : $\Delta \tilde{T}=\Delta T+\Delta T^{\prime}$.
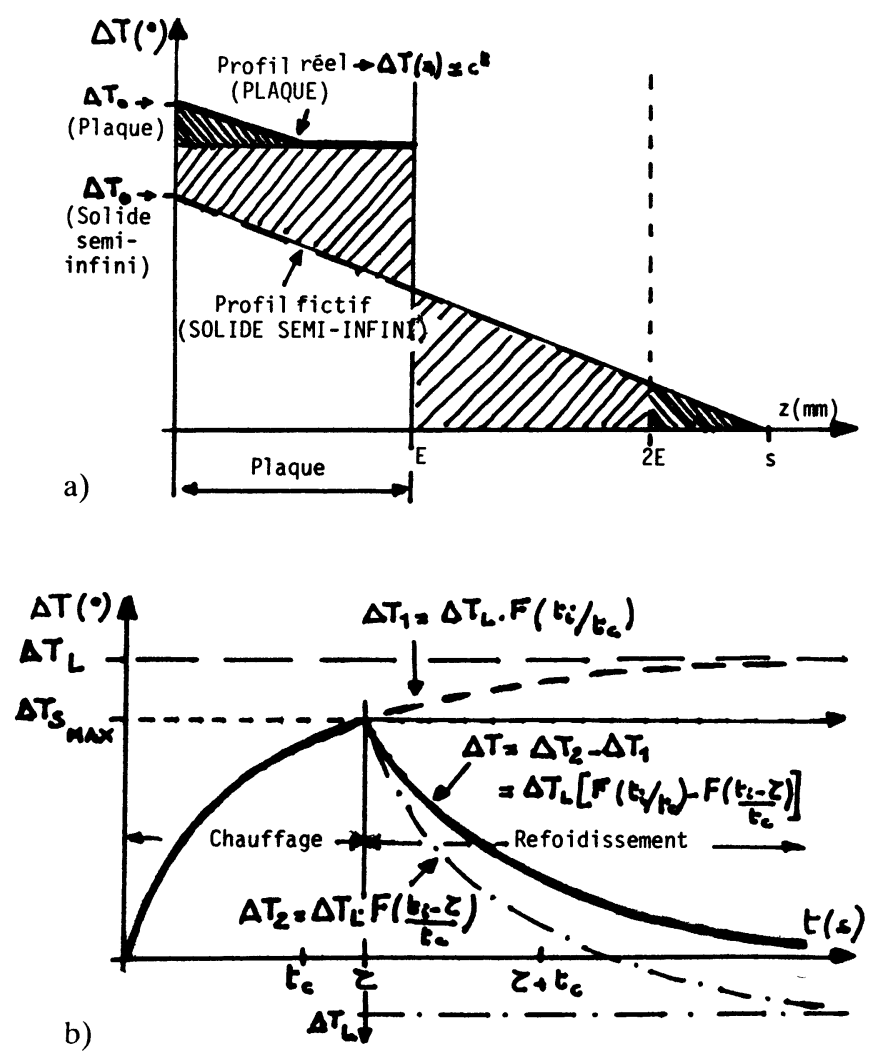

Fig. 5. - a) Principe de la méthode des images appliquée au cas d'une plaque mince. b) Principe de superposition appliqué à la détermination d'une loi de refroidissement.

[a) Principle of the method of images in the case of a thin sheet. b) Principle of superposition applied to the determination of a cooling cycle.]
Par ailleurs notre approche à caractère analytique permet d'utiliser les méthodes de résolution habituellement employées par les thermiciens pour traiter les problèmes de matériaux de dimensions finies (méthode des « images » consistant à considérer que l'énergie est réfléchie sur les parois du matériau voir le schéma figure 5a correspondant à l'irradiation d'une plaque d'épaisseur $E$ ), ou encore pour tenir compte de la modification du flux d'apport énergétique (principe de «superposition" permettant en particulier la description des lois de refroidissement par activation d'une source fictive équivalente et de puissance opposée à partir de l'instant d'extinction $\tau$ - voir schéma figure $5 \mathrm{~b}$ sur lequel au refroidissement $\Delta T_{\mathrm{S}}=\Delta T_{\mathrm{L}}\left\{F\left(t_{\mathrm{i}} / t_{\mathrm{c}}\right)-F\left[\left(t_{\mathrm{i}}-\tau\right) / t_{\mathrm{c}}\right]\right\}$. L'évaluation plus rigoureuse des vitesses de refroidissement nécessaire au métallurgiste devient alors possible et nous consacrerons d'ailleurs un prochain article à ce problème.

Le formalisme que nous proposons dans la présente étude se trouve bien sûr compliqué dès que sont abordées ces situations plus complexes, mais nous insistons sur le fait que les relations de base établies restent valables et que la seule sommation de relations élémentaires de ce type doit permettre de décrire avec une assez bonne approximation la plupart des situations réelles rencontrées par l'expérimentateur et cela même si l'utilisation de microordinateurs s'avère indispensable pour mener à bien ces sommations.

\section{Conclusion.}

La modélisation que nous venons de présenter tient compte des échanges énergétiques en volume dans le matériau, elle entre donc dans la catégorie des modèles dits « tridimensionnels » qui sont habituellement considérés comme nécessairement complexes. Pourtant nous montrons ici qu'il est possible de pratiquer dans ce cadre une analyse simplifiée des effets induits par l'interaction rayonnement lasermatériau, du moins dans le cas d'un apport d'énergie à caractère superficiel, et moyennant certaines adaptations notre démarche serait probablement transposable à d'autres situations. Outre la simplicité de son formalisme, une autre originalité de notre approche est d'essayer de tenir compte des répartitions réelles d'énergie au sein des faisceaux ainsi que de leurs éventuelles fluctuations. Cela est possible grâce à notre approche de type analytique mais aussi et surtout parce que l'on a eu le souci de définir des paramètres pertinents à caractère le plus universel possible pour décrire ces répartitions d'énergie au sein des faisceaux. Bien entendu il serait nécessaire de développer toute une métrologie de la caractérisation des faisceaux à partir de ces suggestions, cela devrait permettre de comparer ou de reproduire des résultats obtenus avec des installations diverses dans 
différents laboratoires ce qui est malheureusement très difficile actuellement.

Si notre approche doit être susceptible de donner accès avec un degré d'incertitude raisonnable (de l'ordre de $10 \%$ ) à des comportements prévisionnels ou à un choix de paramètres de traitements, elle n'est évidemment pas susceptible de décrire dans le détail toutes les évolutions spatiales et temporelles, ni les effets de discontinuités physiques (cas par exemple de matériaux hétérogènes). De ce point de vue une résolution numérique complète d'une équation de la chaleur sera toujours plus performante si les hypothèses de départ et les conditions aux limites sont conformes à la réalité du traitement. Nous insistons sur le fait qu'il n'y a pas d'opposition entre les deux approches. Nous proposons seulement une démarche qui devrait permettre de s'affranchir de la lourdeur de certains calculs conduisant souvent à des résultats aux précisions illusoires compte tenu du manque d'informations que l'on a sur nombre de paramètres; calculs qui sont aussi inadaptés au pilotage en temps réel d'une installation de traitements laser, objectif qu'il nous paraît nécessaire d'avoir présent à l'esprit.

Afin de bien montrer les potentialités de l'approche proposée, tant d'un point de vue de l'application en traitements thermiques que du point de vue de l'analyse des résultats en métallurgie physique, nous l'avons appliquée dans une seconde partie [31] à un certain nombre de situations types.

\section{Annexe 1.}

\section{Caractérisation de faisceaux d'énergie.}

A. DÉFINITION DE PARAMÈTRES CARACTÉRISTIQUES DE FAISCEAUX D'ÉNERGIE. - Soit un faisceau transportant une puissance totale $P$, ayant une section $S$ dans un plan perpendiculaire à sa direction de propagation et possédant dans ce plan une fonction $\psi(x, y)$ de modulation de sa densité de puissance avec une densité de puissance maximale $p_{\mathrm{M}}$; cela implique :

$$
P=p_{\mathrm{M}} \iint_{\delta} \mathrm{d} x \mathrm{~d} y \psi(x, y)
$$

et dans le cas où le faisceau est à symétrie axiale autour de sa direction de propagation :

$$
P=2 \pi p_{\mathrm{M}} \int_{0}^{R} u \mathrm{~d} u \psi(u)
$$

avec $\psi(0)=1$ et $\psi(R)=0$ si le maximum de densité de puissance est effectivement sur la direction de propagation.

Le rayon équivalent $r^{+}$d'un tel faisceau correspond au rayon d'un faisceau cylindrique possédant une densité de puissance maximale constante égale à
$p_{M}$ et transportant la même puissance $P$; on aura donc :

$$
\begin{aligned}
r^{+^{2}} & =\frac{1}{\pi} \iint_{\delta} \mathrm{d} x \mathrm{~d} y \psi(x, y) \\
& =2 \int_{0}^{R} u \mathrm{~d} u \psi(u)=\frac{P}{\pi_{P_{\mathrm{M}}}} .
\end{aligned}
$$

De la même façon il est possible de définir une dimension équivalente $r_{x}$ selon une direction $x$; cette grandeur servira entre autre à l'évaluation des temps d'interaction équivalents pour le cas des sources se déplaçant suivant l'axe $x$ :

$$
r_{x}=\frac{1}{2} \int_{\mathfrak{L}_{1}}^{\mathfrak{f}_{2}} \mathrm{~d} x \psi(x)
$$

$\mathfrak{L}_{1}$ et $\mathfrak{L}_{2}$ étant les limites de définition de $\boldsymbol{\psi}$ dans la direction $x$; ceci conduit, dans le cas d'une symétrie axiale, autour d'un point $O$, à :

$$
r_{\mathrm{o}}=\int_{0}^{R} \mathrm{~d} x \psi(x) .
$$

Cette dimension équivalente peut être évaluée et moyennée dans toutes les directions autour de l'axe du faisceau ce qui conduit à définir $r_{x}$ moyen $\left(\bar{r}_{\mathrm{o}}\right)$, grandeur qui présentera un intérêt du point de vue du comportement thermique.

$$
\bar{r}_{\mathrm{o}}=\frac{1}{2 \pi} \int_{0}^{2 \pi} \mathrm{d} \theta \int_{0}^{\mathfrak{f}(0)} \mathrm{d} u \psi(u, \theta) .
$$

Remarquer que $r_{x}=\bar{r}_{0}$ si le faisceau présente un axe de révolution.

La notion «d'étalement » $E$ peut être introduite en définissant celui-ci comme étant le rapport entre la puissance $P^{+}$transportée par le faisceau à l'intérieur du cylindre de rayon $r^{+}$et la puissance totale $P$ :

$$
E=\frac{P^{+}}{P}=\frac{\int_{0}^{2 \pi} \mathrm{d} \theta \int_{0}^{r^{+}} u \mathrm{~d} u \psi(u, \theta)}{\pi r^{+^{2}}}
$$

De même, on peut définir un facteur d'étalement $g(E)=\bar{r}_{\mathrm{o}} / r^{+}$, grandeur qui conditionne le flux d'échange thermique à l'aplomb du faisceau (voir annexe 2).

\section{B. VALEURS DES DIFFÉRENTS PARAMÈTRES POUR DES RÉPARTITIONS TYPES.}

B.a Répartitions gaussiennes diaphragmées (GD) : figure A.1a. - Considérons une fonction de répartition d'énergie de type gaussienne diaphragmée à un rayon $R=\beta r$ (Fig. A.1a); ceci équivaut à diaphragmer à $\beta_{=}^{+}(1 / e)^{\beta^{2}}$ de la hauteur de la gaussienne, ainsi $\beta=1 \Leftrightarrow 1 / e, \beta=\sqrt{2} \Leftrightarrow 1 / e^{2}, \beta=\sqrt{2} / 2 \Leftrightarrow$ $1 / \sqrt{e}$. De cette façon, toute répartition allant de 


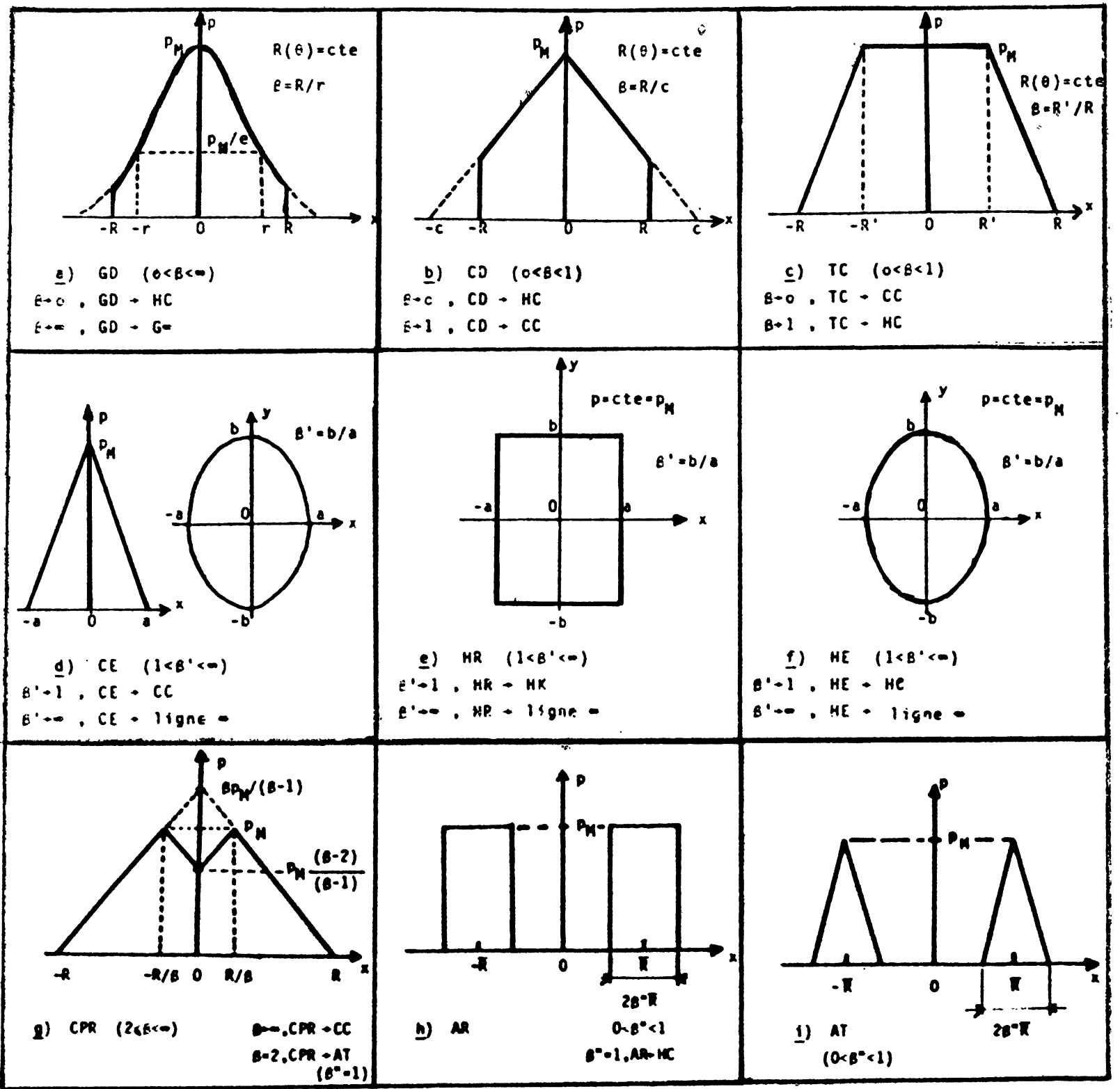

Fig. A.1. - Schémas des répartitions d'énergie.

[Schemas of energy repartitions.]

l'homogène circulaire (HC) à la gaussienne étendue à l'infini $\left(G_{\infty}\right)$ peut être décrite et il peut être montré que :

$$
\begin{gathered}
\psi(u)=\theta(R-u) e^{-u^{2} / r^{2}} \\
r^{+}=r \sqrt{1-e^{-\beta^{2}}} \text { et } \bar{r}_{\mathrm{o}}=\frac{r \sqrt{\pi}}{2} \operatorname{erfc} \beta \\
E=\frac{1-\exp -\left(1-e^{-\beta^{2}}\right)}{1-e^{-\beta^{2}}} \\
g(E)=\frac{\sqrt{\pi} \operatorname{erfc} \beta}{2 \sqrt{1-e^{-\beta^{2}}}} .
\end{gathered}
$$

D'autres répartitions peuvent être envisagées (voir Fig. A.1b-i pour la définition des paramètres décrivant ces répartitions).

B.b Coniques circulaires diaphragmées(CD) : figure A.1b.

$r^{+}=R\left(1-\frac{2}{3} \beta\right)^{1 / 2} \quad$ et $\quad \bar{r}_{\mathrm{o}}=R\left(1-\frac{\beta}{2}\right)$

$E=1-\left(\frac{2}{3} \beta\left(1-\frac{2}{3} \beta\right)^{1 / 2}\right)$

$g(E)=\frac{1-(\beta / 2)}{\sqrt{1-(2 \beta / 3)}}$.

Remarque : $\beta=1$, répartition conique circulaire (C). 
B.c Tronconiques circulaires (TC) : figure A.1c.

$r^{+}=\frac{R}{\sqrt{3}} \sqrt{\beta^{2}+\beta+1}$ et $\bar{r}_{\mathrm{o}}=\frac{R}{2}(1+\beta)$

$E=\frac{1}{1-\beta}\left[1-\frac{\beta^{3}}{\beta^{2}+\beta+1}-\frac{2}{\sqrt{27}} \sqrt{\beta^{2}+\beta+1}\right]$

$g(E)=\frac{\sqrt{3}(1+\beta)}{2 \sqrt{\beta^{2}+\beta+1}}$.

B.d Coniques elliptiques (CE) : figure A.1d.

$r^{+}=\frac{a \sqrt{\beta^{\prime}}}{\sqrt{3}}$ et $\quad \bar{r}_{\mathrm{o}}=\frac{a}{\pi} \nVdash\left(\frac{\sqrt{\beta^{\prime 2}-1}}{\beta^{\prime}}\right)$

$E=1-\frac{4 \sqrt{\beta^{\prime}}}{\pi \sqrt{27}} \cdot \varepsilon\left(\frac{\sqrt{\beta^{\prime 2}-1}}{\beta^{\prime}}\right)$

$g(E)=\frac{\sqrt{3}}{\pi \sqrt{\beta^{\prime}}} \cdot \pi\left(\frac{\sqrt{\beta^{\prime 2}-1}}{\beta^{\prime}}\right)$

avec $\nVdash$ intégrale elliptique complète de première espèce et $\mathcal{E}$ intégrale elliptique complète de deuxième espèce.

B.e Homogènes rectangulaires (HR) : figure A.1e.

et

$$
r^{+}=2 a \sqrt{\beta^{\prime} / \pi}
$$

$$
\bar{r}_{\mathrm{o}}=\frac{2 a}{\pi}\left(\operatorname{lntg}\left(\Omega+\frac{\pi}{4}\right)+\beta^{\prime} \operatorname{lntg}\left(\frac{\pi}{2}-\Omega\right)\right)
$$

avec $\quad \Omega=\frac{1}{2} \operatorname{arctg} \beta^{\prime}$

$E=1-\frac{2 \omega}{\pi}+\frac{1}{\sqrt{\pi \beta^{\prime}}} \sin \omega$

$\operatorname{avec} \omega=\operatorname{arcos}\left(\frac{1}{2} \cdot \sqrt{\frac{\pi}{\beta^{\prime}}}\right)$

$g(E)=\frac{1}{\sqrt{\pi \beta^{\prime}}}\left(\operatorname{lntg}\left(\Omega+\frac{\pi}{4}\right)+\right.$

$$
\left.+\beta^{\prime} \operatorname{lntg}\left(\frac{\pi}{2}-\Omega\right)\right) \text {. }
$$

Remarque : $\beta^{\prime}=1$, répartition homogène carrée (HK).

B.f Homogènes elliptiques (HE) : figure A.1f.

$r^{+}=a \sqrt{\beta^{\prime}}$ et $\bar{r}_{\mathrm{o}}=\frac{2 a}{\pi} \nVdash\left(\frac{\sqrt{\beta^{\prime 2}-1}}{\beta^{\prime}}\right)$

$E=1-\frac{2}{\pi}\left[\operatorname{arctg} \sqrt{\beta^{\prime}}-\operatorname{arctg} \frac{1}{\sqrt{\beta^{\prime}}}\right]$

$g(E)=\frac{2}{\pi \sqrt{\beta^{\prime}}} \pi\left(\frac{\sqrt{\beta^{\prime 2}-1}}{\beta^{\prime}}\right)$ avec $\pi$ intégrale elliptique complète de première espèce.

B.g Coniques à pointe rentrée (CPR) : figure A.1g.

$r^{+}=\frac{R}{\beta \cdot \sqrt{3}} \cdot \sqrt{\frac{\beta^{3}-2}{\beta-1}}$ et $\bar{r}_{\mathrm{o}}=\frac{R}{2} \cdot \frac{\beta^{2}-2}{\beta(\beta-1)}$

$E=\frac{\beta}{\beta-1}-\frac{2}{\beta^{3}-2}-\frac{2 \sqrt{\beta^{3}-2}}{3^{3 / 2}(\beta-1)^{3 / 2}}$

$g(E)=\frac{\sqrt{3}\left(\beta^{2}-2\right)}{2 \sqrt{(\beta-1)\left(\beta^{3}-2\right)}}$.

B.h Annulaires "rectangulaires » (AR): figure A.1h.

$r^{+}=2 \bar{R} \sqrt{\beta^{\prime \prime}} \quad$ et $\quad \bar{r}_{\mathrm{o}}=2 \bar{R}_{\beta^{\prime \prime}}$

$E=1-\frac{\left(1-\beta^{\prime \prime}\right)^{2}}{4 \beta^{\prime \prime}}$

$g(E)=\sqrt{\beta^{\prime \prime}}$.

B.i Annulaires «triangulaires» (AT) : figure A.1i.

$r^{+}=\bar{R} \sqrt{2 \beta^{\prime \prime}}$ et $\bar{r}_{\mathrm{o}}=\bar{R} \beta^{\prime \prime}$

$\left(2-\sqrt{3}<\beta^{\prime \prime}<\frac{1}{2}\right) \Rightarrow E=\frac{3}{2}\left(1-\frac{1}{\beta^{\prime \prime}}\right)+$ $+\frac{1}{6}\left(\frac{1}{\beta^{\prime \prime 2}}-\beta^{\prime \prime}\right)+\frac{2^{3 / 2}}{3 \sqrt{\beta^{\prime \prime}}}$

$\left(\frac{1}{2}<\beta^{\prime \prime}<1\right) \Rightarrow E=\frac{3}{2}\left(1-\frac{1}{\beta^{\prime \prime}}\right)-$

$-\frac{1}{6}\left(\beta^{\prime \prime}+\frac{1}{\beta^{\prime \prime 2}}\right)-\frac{2^{3 / 2}}{3 \sqrt{\beta^{\prime \prime}}}$

$g(E)=\frac{\sqrt{\beta^{\prime \prime}}}{\sqrt{2}}$.

C. DÉtermination EXPÉRIMENTALE DE $\bar{r}_{0} \cdot-\mathrm{Si}$ on réalise la mesure de la puissance $P_{\varepsilon}$ que laisse passer une fente $\mathcal{F}_{\varepsilon}$ orientée selon la direction $\mathrm{O} x$ et de largeur $\varepsilon$ dans la direction $y$ (Fig. A.2), il est possible d'écrire :

$$
P_{\varepsilon}=p_{\mathrm{M}} \int_{\mathcal{F}} \int_{\varepsilon} \mathrm{d} x \mathrm{~d} y \psi(x, y)
$$

et si l'on admet que la fente est suffisamment étroite pour que la répartition ne varie pas dans la direction $y$, on peut écrire :

$$
\psi(x, y)=\psi_{x}(x) . \psi_{y}(y) \simeq \psi_{x}(x)
$$

d'où il vient

$$
P_{\varepsilon}=\varepsilon p_{\mathrm{M}} \int_{\mathfrak{L}_{1}}^{\mathfrak{C}_{2}} \psi_{x}(x) \mathrm{d} x=2 r_{x} \varepsilon p_{\mathrm{M}} .
$$




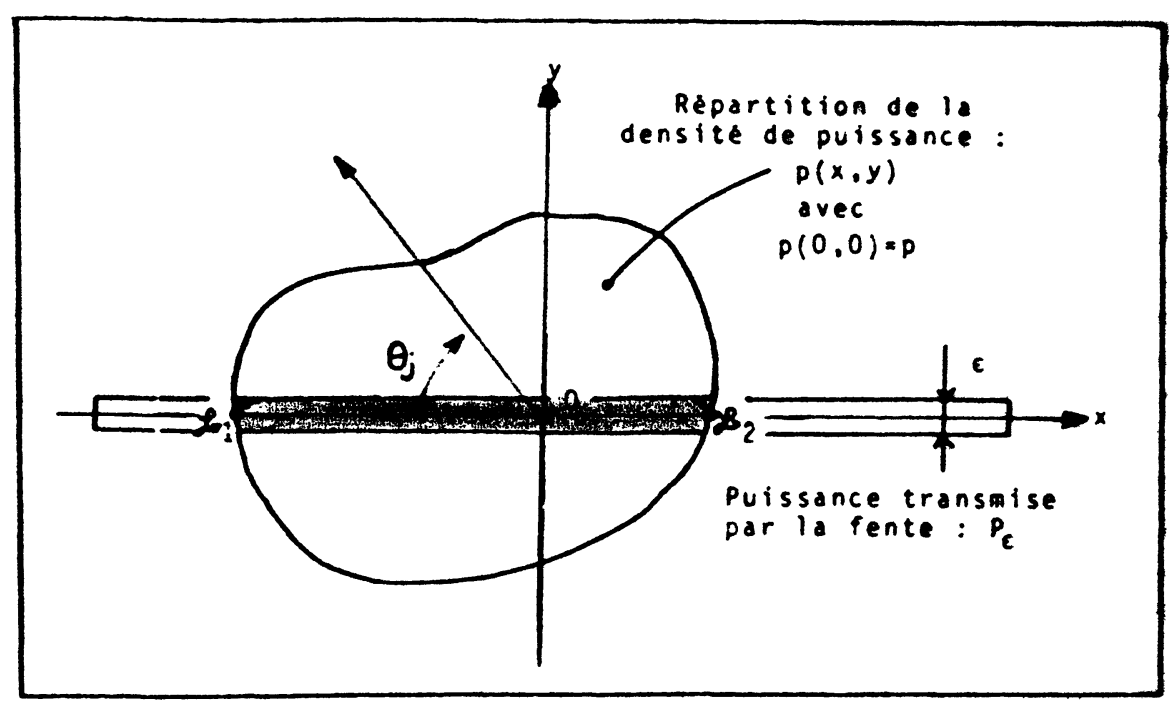

Fig. A.2. - Principe de détermination expérimentale de $\bar{r}_{0}$ (méthode des fentes).

[Principle of the experimental determination of $\bar{r}_{\mathrm{o}}$ (slit method).]

En répétant cette opération pour $n$ positions de la fente (angles $\boldsymbol{\theta}_{j}$ ), on peut moyenner les mesures de $P_{\varepsilon_{j}}$ et par conséquent :

$$
\bar{r}_{\mathrm{o}}=\frac{\sum_{j=1}^{n} P_{\varepsilon_{j}}}{2 n \varepsilon p_{M}} .
$$

\section{Annexe 2.}

\section{Evaluation des champs thermiques.}

A. Solution analytiQue générale de l'ÉQuaTION DE LA CHALEUR. - Considérons une équation linéaire de transfert de chaleur dans un milieu isotrope infini

$$
\left(\frac{\partial}{\partial t}-\alpha \nabla^{2}\right) \Delta T(r, t)=\frac{\alpha}{k} f(r, t) .
$$

$\Delta T$ représente au point $r=(x, y, z)$ et à l'instant $t$ l'accroissement de température résultant de la génération $f(r, t) ; \alpha$ et $k$ sont respectivement diffusivité et conductivité thermiques du matériau.

La résolution de cette équation peut s'effectuer en calculant la fonction de Green $\mathcal{G}\left(r, t / r^{\prime}, t^{\prime}\right)$ de l'opérateur, fonction définie par

$$
\left(\frac{\partial}{\partial t}-\alpha \nabla^{2}\right) \mathcal{G}\left(r, t / r^{\prime}, t^{\prime}\right)=\delta\left(r-r^{\prime}\right) \delta\left(t-t^{\prime}\right)
$$

et qui représente l'effet au point $r$ et à l'instant $t$ d'une source ponctuelle émettant à l'instant $t^{\prime}$ et au point $r^{\prime}$ une quantité unitaire d'énergie.

Pour un solide semi-infini limité par un plan d'abscisse $z=0 \mathrm{G}$ s'exprimera par :

$$
\mathcal{G}\left(r, t / r^{\prime}, t^{\prime}\right)=\frac{\exp -\left[\left(r-r^{\prime}\right)^{2} / 4 \alpha\left(t-t^{\prime}\right)\right]}{8\left[\pi \alpha\left(t-t^{\prime}\right)\right]^{3 / 2}}
$$

En décomposant $f(r, t)$ en combinaison linéaire de sources ponctuelles, l'équation de départ peut être intégrée :

$$
\Delta T(r, t)=\frac{\alpha}{k} \int_{\smile} \mathrm{d} t^{\prime} \int_{\mathcal{V}} \mathrm{d} v^{\prime} \times \mathcal{G}\left(r, t / r^{\prime}, t^{\prime}\right) f\left(r^{\prime}, t^{\prime}\right)
$$

où $\mathfrak{C}$ et $\vartheta$ sont les étendues temporelles et spatiales sur lesquelles l'émission de chaleur a lieu dans le solide.

Si celle-ci se produit en surface (plan $z=0$ ), sur une aire $\delta$, avec une densité de puissance maximale $p_{\mathrm{M}}$ et un rendement d'absorption $\rho$,

$$
f\left(r^{\prime}, t^{\prime}\right)=\rho p_{M} \cdot \delta\left(z^{\prime}\right) \psi\left(x^{\prime}, y^{\prime}, t^{\prime}\right)
$$

et

$$
\begin{aligned}
& \Delta T(r, t)=\frac{\rho p_{\mathrm{M}}}{4 \pi^{3 / 2} k \sqrt{\alpha}} \times \\
& \times \int_{\mathcal{C}} \frac{\mathrm{d} t^{\prime}}{\left(t-t^{\prime}\right)^{3 / 2}} \exp -\frac{z^{2}}{4 \alpha\left(t-t^{\prime}\right)} \\
& \quad \times \iint_{\delta\left(t^{\prime}\right)} \mathrm{d} x^{\prime} \mathrm{d} y^{\prime} \times \\
& \quad \times \exp \left[-\frac{\left(x-x^{\prime}\right)^{2}+\left(y-y^{\prime}\right)^{2}}{4 \alpha\left(t-t^{\prime}\right)}\right] \psi\left(x^{\prime}, y^{\prime}, t^{\prime}\right) .
\end{aligned}
$$

B. CAS D'Un faisceau fiXe. - En éclairant le solide à partir du temps $t=0$ le spot étant fixe et en choisissant un repère dont l'origine coïncide avec le maximum de densité de puissance, l'accroissement 
de température $\Delta T$ (à l'origine du repère) s'exprime en coordonnées cylindriques par :

$$
\begin{aligned}
\Delta T=\frac{\rho p_{\mathrm{M}}}{4 \pi^{3 / 2} k \sqrt{\alpha}} \int_{0}^{t} \frac{\mathrm{d} \omega}{\omega^{3 / 2}} \int_{0}^{2 \pi} \mathrm{d} \theta \times \\
\quad \times \int_{0}^{\mathcal{L}(\theta)} \nu \mathrm{d} \nu \psi(\nu, \theta) \exp -\frac{\nu^{2}}{4 \alpha \omega}
\end{aligned}
$$

où $\omega=t-t^{\prime}$ et $\mathcal{L}(\theta)$ est la limite de la répartition d'énergie exprimée en coordonnées polaires.

Deux cas sont a considérer selon que les temps d'éclairement sont très brefs ou très longs :

B.a Temps très courts (régime transitoire). - En posant $\frac{\nu^{2}}{4 \alpha \omega}=u$ la troisième intégrale de l'expression (A.9) se transforme :

$$
\begin{aligned}
\Delta T=\frac{\rho p_{\mathrm{M}} \sqrt{\alpha}}{2 k \pi^{3 / 2}} & \int_{0}^{t} \frac{\mathrm{d} \omega}{\sqrt{\omega}} \int_{0}^{2 \pi} \mathrm{d} \theta \times \\
& \times \int_{0}^{\mathcal{L}^{2}(\theta) / 4 \alpha \omega} \mathrm{d} u \psi(2 \sqrt{\alpha u \omega}, \theta) e^{-u} .
\end{aligned}
$$

Or, puisque $\omega$ reste très petit seule la valeur de $\psi$ au voisinage de l'origine est à considérer, et de plus la borne supérieure de cette troisième intégrale est très grande, d'où :

$\int_{0}^{\mathfrak{L}^{2}(\theta) / 4 \alpha \omega} \mathrm{d} u \psi(2 \sqrt{\alpha u \omega}, \theta) \cdot e^{-u} \simeq \int_{0}^{\infty} \mathrm{d} u e^{-u}=1$.

Le résultat final pour ce régime sera :

$$
\Delta T=\frac{2 \rho p_{\mathrm{M}} \sqrt{\alpha t}}{k \sqrt{\pi}}=\frac{2 \rho P \sqrt{\alpha t}}{k r^{+^{2}} \pi^{3 / 2}}
$$

$\left(r^{+}\right.$rayon équivalent du faisceau dans la zone d'interaction, d'après (A.2)).

B.b Temps très longs (régime quasi stationnaire). Dans l'expression (A.9) les intégrations peuvent se permuter :

$$
\begin{array}{r}
\Delta T=\frac{\rho p_{\mathrm{M}}}{4 k \sqrt{\alpha} \pi^{3 / 2}} \int_{0}^{2 \pi} \mathrm{d} \theta \int_{0}^{\mathfrak{L}(\theta)} \nu \mathrm{d} \nu \psi(\nu, \theta) \times \\
\times \int_{0}^{\infty} \frac{\mathrm{d} \omega}{\omega^{3 / 2}} \exp -\frac{\nu^{2}}{4 \alpha \omega}
\end{array}
$$

et en posant dans la dernière intégrale :

$$
\frac{\nu^{2}}{4 \alpha \omega}=\mathcal{G}^{2}
$$

on obtient :

$$
\Delta T=\frac{\rho p_{\mathrm{M}}}{2 k \pi} \int_{0}^{2 \pi} \mathrm{d} \theta \int_{0}^{\mathfrak{L}(\theta)} \mathrm{d} \nu \psi(\nu, \theta)
$$

d'où, d'après (A.5) : $\Delta T=\Delta \mathrm{T}_{\mathrm{L}}=\frac{\rho p_{\mathrm{M}} \bar{r}_{\mathrm{o}}}{k}$, indépendamment du temps.

Cette dernière relation entraîne

$$
\Delta T_{\mathrm{L}}=\frac{\rho P}{k r^{+} \pi} g(E)
$$

avec $g(E)=\bar{r}_{\mathrm{o}} / r^{+}$(conformément à la définition de l'annexe 1).

\section{Remarques :}

1) L'égalisation des expressions (A.10) et (A.11) permet de définir un temps $t_{\mathrm{c}}$, qui sera appelé temps critique :

$$
t_{\mathrm{c}}=\left[\pi r^{+^{2}} g(E)^{2}\right] / 4 \alpha=\pi \bar{r}_{\mathrm{o}}^{2} / 4 \alpha .
$$

2) Comme nous le verrons en annexe 4 , la notion $g(E)$ peut être généralisée en tout point du plan d'interaction, ce qui permettra de remonter aux accroissements superficiels de température, observables en régime «stationnaire » en ces points.

B.c Définition d'un régime intermédiaire. - Pour des temps d'interaction de l'ordre de $t_{c}$, les limites asymptotiques ne permettent pas une évaluation des grandeurs utiles avec une précision suffisante et les

\begin{tabular}{|c|c|c|c|c|c|c|}
\hline Répartition & \multicolumn{3}{|c|}{$\mathrm{HC}: g(E)=1$} & \multicolumn{3}{|c|}{$G_{\infty}: g(E)=\sqrt{\pi} / 2$} \\
\hline Valeurs de $t_{\mathrm{i}}$ & $t^{\prime}$ & $t_{\mathrm{c}}$ & $t^{\prime \prime}$ & $t^{\prime}$ & $t_{\mathrm{c}}$ & $t^{\prime \prime}$ \\
\hline Valeurs exactes de $\Delta T / \Delta T_{\mathrm{L}}$ & 0,47 & 0,70 & 0,85 & 0,29 & 0,64 & 0,87 \\
\hline Approx. $\Delta T / \Delta T_{\mathrm{L}}=\left[g(E)^{8} / \sqrt{2}\right]\left(t_{\mathrm{i}} / t_{\mathrm{c}}\right)^{1 / 4}$ & 0,50 & 0,71 & 1 & 0,31 & 0,57 & 1 \\
\hline Erreurs & $+6 \%$ & $+2 \%$ & $+17 \%$ & $+7 \%$ & $-14 \%$ & $+14 \%$ \\
\hline
\end{tabular}
expressions générales n'étant évaluables que numériquement nous avons été amenés à rechercher dans quelle mesure une expression approchée est susceptible de décrire le comportement dans cette zone « intermédiaire ». On a pu montrer qu'une loi en $\left(t_{\mathrm{i}} / t_{\mathrm{c}}\right)^{1 / 4} \mathrm{~s}$ 'applique raisonnablement sur un intervalle $\left(t^{\prime}, t^{\prime \prime}\right)$ défini à partir de $g(E)$ et de $t_{\mathrm{c}}$ : $t^{\prime}=\left[g(E)^{8} / 4\right] t_{\mathrm{c}}$ et $t^{\prime \prime}=\left[4 / g(E)^{8}\right] t_{\mathrm{c}}$. Quelques résultats sont rassemblés dans le tableau ci-dessous ; on constate que l'approximation proposée conduit à 
des erreurs $\simeq 10 \%$ (les erreurs maximales observables correspondent à $t=t^{\prime}, t_{\mathrm{c}}$ et $\left.t^{\prime \prime}\right)$.

B.d Conjecture. - Diverses conclusions présentées dans la suite reposent sur une conjecture suivant laquelle une fois $r_{\mathrm{o}}^{+}$et $E$ fixés (c'est-à-dire $p_{0}, P$ et $P^{+}$mesurés) le comportement thermique est pratiquement indépendant du choix de la fonction de modulation de la densité de puissance si cette modulation présente un maximum central. Les diagrammes $(P, t)$ et $(\Delta T, t)$ pourront donc être établis en considérant les résultats des gaussiennes diaphragmées. Nous avons pu vérifier que cette conjecture conduit à des approximations de l'ordre de quelques $\%$ pour plusieurs répartitions types (voir annexe 3 ), précision suffisante étant données les incertitudes existant sur l'ensemble des caractéristiques du matériau et de l'interaction laser-matériau.

C. CAS D'Un faisceau mobile. - Dans le cas d'une répartition axisymétrique se déplaçant à la vitesse $v$ et ayant une dimension de $2 r_{x}$ selon cette direction de déplacement, le faisceau peut être considéré comme délimité par un front avant et un front arrière équivalents, respectivement distants de $r_{x}$ et $-r_{x}$ de son axe de propagation. En un point particulier de la direction de déplacement, en surface du matériau, l'origine des temps $(t=0)$ sera choisie à l'instant du passage du front équivalent avant.

Le temps d'éclairement équivalent $t^{+}$vaut $r_{x} / v$ lorsque l'axe du faisceau et le point coïncident et au passage du front arrière, $t^{+}=2 r_{x} / v=\tau^{+}$, temps d'interaction équivalent.

Deux régimes sont à distinguer selon la valeur de la vitesse.

C.a Vitesses très faibles. - Le comportement permanent des faisceaux fixes se retrouve. Le maximum d'accroissement de température $\Delta T_{\mathrm{L}}$ est en regard de celui de densité de puissance et est indépendant du temps d'interaction, donc de la vitesse de déplacement.

A l'arrière de la zone d'interaction, on atteint également une température limite pouvant être déterminée en tenant compte de $g(E)_{r_{x}}$, observé à la distance $r_{x}$ de l'axe du faisceau (voir annexe 4).

C.b Vitesses élevées. - Il existe un régime transitoire durant lequel la température évolue en $v^{-1 / 2}$, le front arrière équivalent correspondant au maximum de température, c'est-à-dire, à un temps d'éclairement équivalent $2 r_{x} / v$. Celui-ci étant le double de celui observé dans l'axe du faisceau à un instant donné, la température «arrière » sera plus élevée d'un facteur $\sqrt{2}$ que celle en regard de l'axe.

L'intersection des limites asymptotiques des températures maximales observées dans les deux régimes conduit à définir une vitesse critique $v_{\mathrm{c}}$. Si l'on effectue le changement de variable $v=2 r_{x} / \tau^{+}$on constate qu'il y a correspondance entre $v_{\mathrm{c}}$ et le temps critique $t_{\mathrm{c}}$ qui serait observé avec le même faisceau fixe. Par conséquent $v_{\mathrm{c}} t_{\mathrm{c}}=2 r_{x}$ et :

$$
v_{\mathrm{c}}=(8 \alpha / \pi) r_{x} / \bar{r}_{\mathrm{o}}^{2}=(8 \alpha / \pi) r_{x} / g(E)^{2} r^{+^{2}} \text {. }
$$

Lorsque le faisceau présente un axe de révolution, ces expressions se simplifient $\left(r_{x}=\bar{r}_{\mathrm{o}}\right): v_{\mathrm{c}}=$ $8 \alpha / \pi \bar{r}_{\mathrm{o}}=8 \alpha / \pi g(E) r^{+}$.

Des courbes isothermes et isoflux avec la vitesse comme variable pourront donc être déduites de celles concernant la même répartition en faisceau fixe. Il faut toutefois remarquer que ces courbes permettront de déterminer la valeur maximale de la température atteinte sans préciser la localisation de cet extremum. Par ailleurs, le régime intermédiaire se trouvera plus étendu que dans le cas des faisceaux fixes; on prendra $v^{\prime}=\left[6 / g(E)^{8}\right] v_{c}$ et $v^{\prime \prime}=$ $\left[g(E)^{8} / 6\right] v_{\mathrm{c}}$ ce qui correspond à $t^{\prime+}=2 t^{\prime} / 3$ et $t^{\prime \prime}=3 t^{\prime \prime} / 2$.

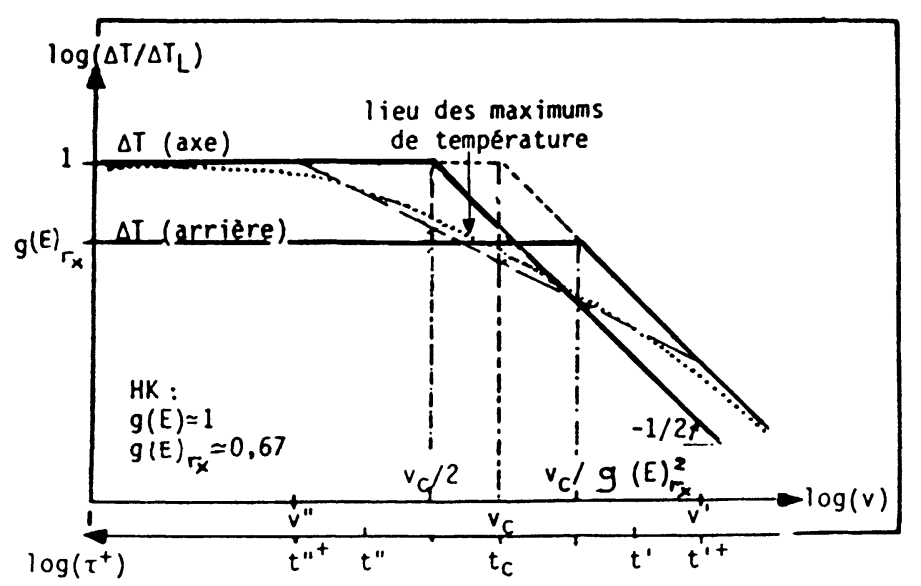

Fig. A.3. - Diagramme «isoflux » représentant $\Delta T$ en fonction de v pour une source homogène carrée mobile. [«Isoflux » diagram showing $\Delta T$ with $v$ for a homogeneous square moving repartition.] 
La figure A.3 illustre ces propos dans le cas d'une source homogène carrée HK pour laquelle $r_{x}=a$ (demi-côté du carré), $\bar{r}_{\mathrm{o}} \simeq 1,12 a, r^{+} \simeq 1,13 a$, $g(E) \simeq 0,995$ et $g(E)_{r_{x}}=0,68 g(E) \simeq 0,67$ (d'après annexe 4).

\section{Annexe 3.}

Relations entre facteur de forme et étalement.

ÉVALUATION DE LA FONCTION $g(E)$ A PARTIR DE L'ÉTALEMENT $E$. - La conjecture proposée en annexe 2 est illustrée ici, en traçant $g(E)$ en fonction de $E$ (Fig. A.4a), pour les répartitions décrites en annexe 1. Tous les résultats correspondent à une

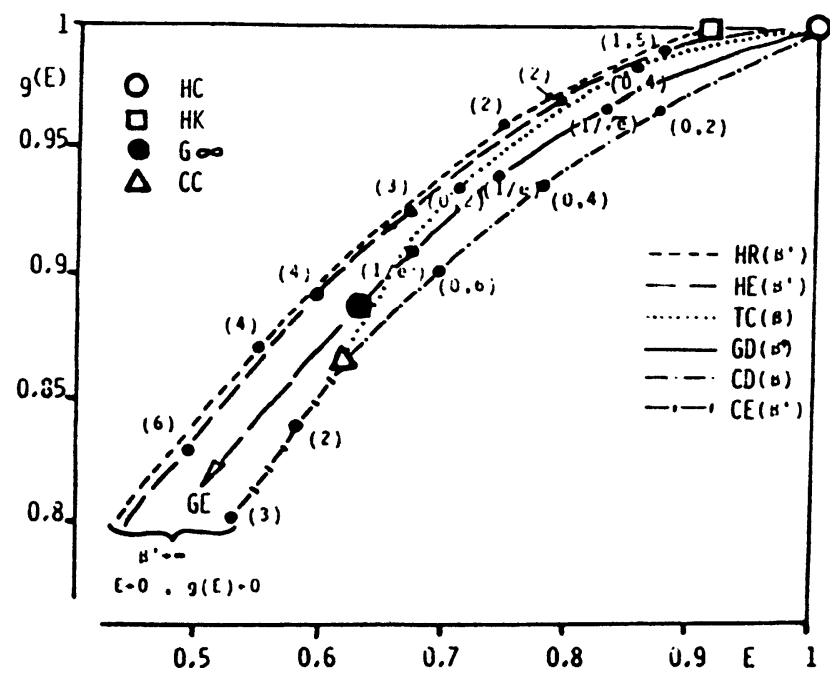

a)

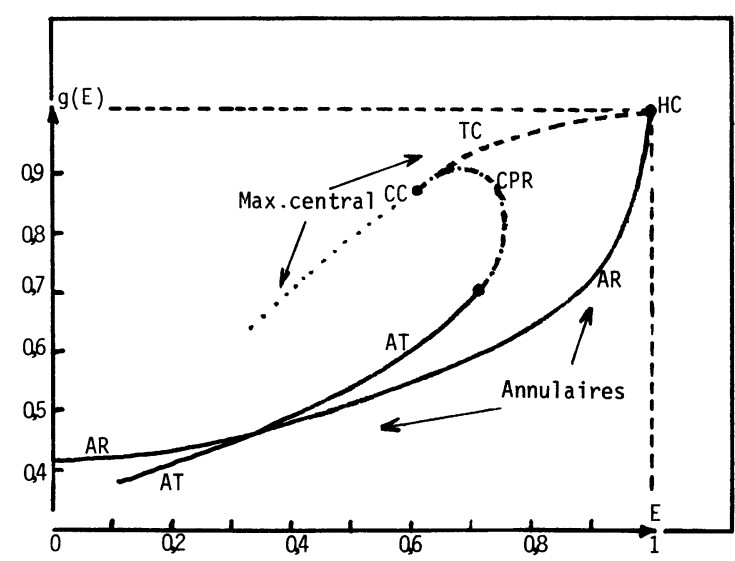

b)

Fig. A.4. - a) Représentation de $g(E)$ en fonction de $E$ pour illustrer la conjecture proposée pour les faisceaux à maximum central. b) Possibilité d'une autre conjecture pour les faisceaux de type annulaire.

(a) Representation of $g(E)$ with $E$ as an illustration of the conjecture suggested for beams with a central maximum.

b) Another conjecture of the same type may exist in the case of annular beams.] même puissance transportée $P$ et à une même densité de puissance maximale $p_{M}$ donc s'appliquent à des faisceaux ayant même rayon équivalent $r^{+}$.

A partir du moment où une répartition d'énergie présente un maximum central, la figure A.4a montre que quelle que soit cette répartition, une évaluation expérimentale de $E$ permettra de déterminer une valeur de $g(E)$ ne s'écartant pas de plus de quelques $\%$ de celle conditionnant le traitement thermique. De plus, pour un faisceau axisymétrique réel des déterminations expérimentales de $E$ et $r^{+}$permettront de le décrire par une répartition gaussienne équivalente. Pour $0,63<E<1$, cette répartition serait de type gaussienne diaphragmée circulaire, avec un rapport $\beta$ (voir définition dans annexe 1) variable $(\infty>\beta>0)$; pour $E<0,63$, la répartition équivalente serait de type gaussienne elliptique illimitée $(G E \infty)$ de rapport $\beta^{\prime}$ variable $\left(1<\beta^{\prime}<\infty\right)$.

Le même type de conjecture peut être émis pour les sources annulaires, comme l'illustre la figure A.4b qui représente $g(E)$ en fonction de $E$ pour des sources à répartition annulaire «triangulaire » et annulaire « rectangulaire ».

On doit remarquer que des répartitions ayant des formes assez nettement différentes (par exemple de tronconique $-\mathrm{TC}(0<\beta<0,2)-$ à conique à pointe rentrée - CPR $(1<\beta<\infty)$ - en passant par la conique circulaire $-\mathrm{CC}-$, voir annexe 1$)$, possèdent un facteur d'étalement central $g(E)$ très voisin $(0,85 \pm 0,05)$, comme cela apparaît sur la figure A.4b. Par suite en régime stationnaire les températures limites atteintes dans l'axe du faisceau seront voisines, toutes choses étant égales par ailleurs. En particulier certaines fluctuations dans la répartition d'énergie pourront très bien n'avoir de ce fait qu'une très faible incidence au niveau des effets induits ; nous l'avons constaté dans le cas d'un laser du type CILAS CI 4000 [26].

\section{Annexe 4.}

Généralisation des paramètres caractéristiques d'un faisceau.

A. GÉnéralisation DE $g(E)$. - La notion $g(E)$ peut être généralisée en tout point $\Omega$ du plan d'interaction. On écrit pour cela qu'au point $\Omega$, $g(E)_{\Omega}$ est égal à $\bar{r}_{\Omega} / r^{+}$où $\bar{r}_{\Omega}$ est la moyenne, vue de $\Omega$, des distances équivalentes de la répartition d'énergie. Cette notion permet d'accéder au champ de température en surface du matériau en régime stationnaire en introduisant $G_{\Omega}=g(E)_{\Omega} / g(E)=$ $\bar{r}_{\Omega} / \bar{r}_{\mathrm{o}}$ et $\Delta T_{\mathrm{L}}(\Omega)=G_{\Omega} \cdot \Delta T_{\mathrm{L}}$, (avec $\Delta T_{\mathrm{L}}$ température limite atteinte dans l'axe du faisceau, conformément à (A.11)).

Ainsi dans le cas d'une source homogène de 


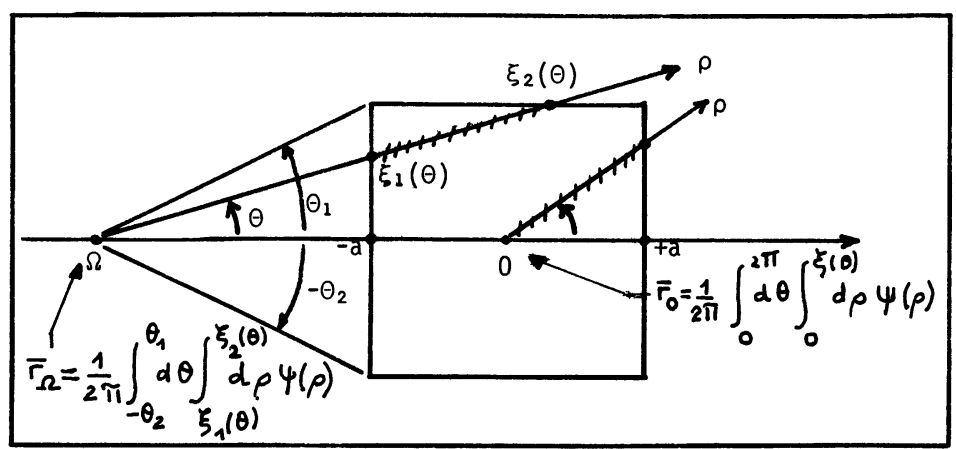

Fig. A.5. - Schéma et principe de calcul du facteur $\bar{r}$ aux points 0 et $\Omega$ pour un faisceau de section carrée.

[Schema and principle of the determination of $\bar{r}$ factor at 0 and $\Omega$ points for a square section beam.]

section carrée de côté $a$ (voir Fig. A.5) il est possible de démontrer:

$$
\begin{aligned}
\frac{\Delta T_{\mathrm{L}}(\Omega)}{\Delta T_{\mathrm{L}}}=\frac{1}{A} & \left\{\operatorname{lntg}\left(\frac{\operatorname{arctg}(j+1)}{2}+\frac{\pi}{4}\right)+\right. \\
& +(j+1) \operatorname{lntg}\left(\frac{\pi}{2}-\frac{\operatorname{arctg}(j+1)}{2}\right) \\
& +\operatorname{lntg}\left(\frac{\operatorname{arctg}(1-j)}{2}+\frac{\pi}{4}\right) \\
& \left.+(1-j) \operatorname{lntg}\left(\frac{\pi}{2}-\frac{\operatorname{arctg}(1-j)}{2}\right)\right\}
\end{aligned}
$$

avec $A=4 \ln (\operatorname{tg}(3 \pi / 8))$ et $j=\mathrm{O} \Omega / a$ ce qui conduit au tableau suivant :

\begin{tabular}{|c|l|l|l|l|}
\hline$j$ & 0 & 0,5 & 1 & 2 \\
\hline$\Delta T_{\mathrm{i}}(\Omega) / \Delta T_{\mathrm{L}}$ & 1 & 0,93 & 0,68 & 0,29 \\
\hline
\end{tabular}

d'où le profil présenté sur la figure A.6.

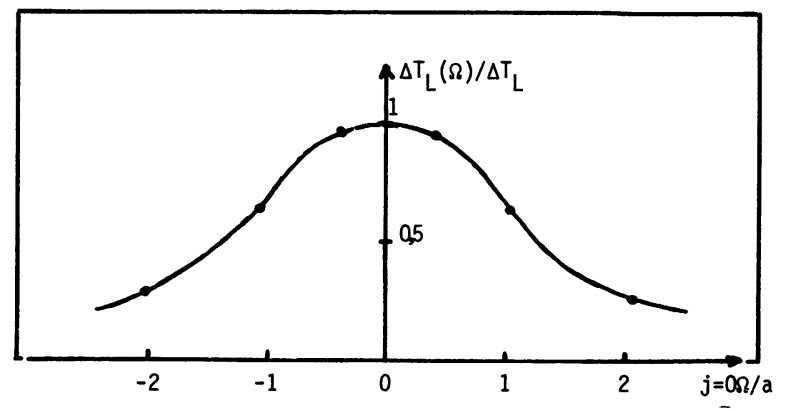

Fig. A.6. - Profil thermique en surface pour une répartition homogène de section carrée en régime stationnaire.

[Surface thermal profile for an homogeneous square repartition, in stationnary behaviour.]

B. DÉTERmination eXPérimentale de $G_{\Omega}$. En plaçant une fente de largeur $\varepsilon$ dans la direction $(\Omega, \rho)$ et en mesurant la puissance $P^{\prime}(\Omega, \theta)$ qu'elle transmet, on peut déduire $r_{x}(\Omega, \theta)$ pourvu que $\varepsilon$ soit suffisamment faible :

$$
r_{x}(\Omega, \theta)=\frac{P^{\prime}(\Omega, \theta)}{2 \varepsilon p_{M}} .
$$

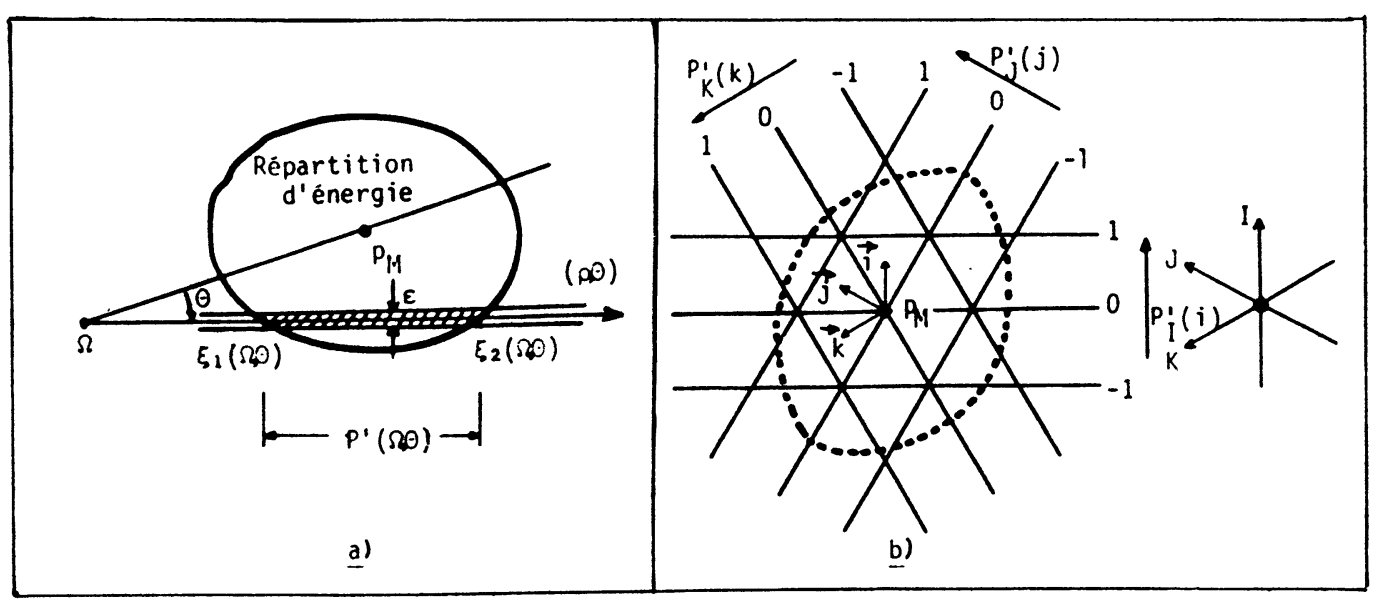

Fig. A.7. - Application de la méthode des fentes à la détermination de : a) $\bar{r}_{\Omega}$ et b) $G_{\Omega}$.

[Application of the slit method in the determination of : a) $\bar{r}_{\Omega}$ and b) $G_{\Omega}$. 
En réalisant $n$ mesures avec des angles $\theta_{i}$ divers, on aura :

$$
\bar{r}_{\Omega}=\frac{1}{2 n \varepsilon p_{\mathrm{M}}} \sum_{i=1}^{n} P_{\mathrm{i}}^{\prime}\left(\Omega, \theta_{\mathrm{i}}\right) .
$$

Définissons maintenant, dans un plan perpendiculaire à l'axe de propagation, trois directions $I, J$ et $K$ à $60^{\circ}$ l'une de l'autre et trois vecteurs unitaires $\mathbf{i}, \mathbf{j}$ et k correspondants (Fig. A.11b). Réalisons ensuite trois séries de mesures de puissance $P_{I}^{\prime}(i), P_{J}^{\prime}(j)$ et $P_{K}^{\prime}(k)$, en translatant la fente successivement dans chaque direction d'une quantité donnée. On peut alors en chaque point $\Omega(i, j, k)$ tel que $\mathbf{O} \Omega=$ $i \cdot \mathbf{i}+j \cdot \mathbf{j}+k \cdot \mathbf{k}$ évaluer $\bar{r}_{\Omega}$ par :

$$
\begin{gathered}
\bar{r}_{\Omega}=\left[P_{I}^{\prime}(i)+P_{J}^{\prime}(j)+P_{K}^{\prime}(k)\right] / 6 \varepsilon p_{\mathrm{M}} \\
G_{\Omega}=\frac{P_{I}^{\prime}(i)+P_{J}^{\prime}(j)+P_{K}^{\prime}(k)}{P_{I}^{\prime}(0)+P_{J}^{\prime}(0)+P_{K}^{\prime}(0)}
\end{gathered}
$$

et

On obtient ainsi une information sur le champ de température en régime stationnaire en surface d'un solide semi-infini.

\section{References}

[1] Miller, J. E. and Wineman, J. A., Metal Progress 11 (1977) 38

[2] Yessik, M. S., Opt. Eng. 17 (1978) 202.

[3] Gnanamuthu, D. S., Appl. of Lasers in Material Processing, A.S.M. (1979) 177.

[4] MaZumder, J., J. Met. 35 (1983) 18.

[5] Bloembergen, N., Appl. of Lasers in Material Processing, A.S.M. 1 (1979).

[6] Steen, W. N. and Courtney, C., Met. Techn. 12 (1979) 456.

[7] Zhang Guangyum et al., Proceed. of 3rd Int. Congress on Heat Treatment of Materials, Shanghaï, Ed. Metals Soc., London, 2.9 (1984).

[8] Chamde, T. and Mazumder, J., Lasers in Metallurgy, Metal. Soc. of AIME, 165 (1981).

[9] Gnanamuthu, D. S. and Shankar, S., Laser Surface Treatment of Metals, Ed. W. Draper and P. Mazzoldi (Martinus Nijhoff Publish.) 413 (1986).

[10] Kechemair, D. and Gerbet, D., Proc. L.I.M. 3, Ed. Kenzer (I.F.S. Publ.) 261 (1986).

[11] Giovanola, B., Thèse $n^{\circ} 647$, E.P.F. Lausanne (1986).

[12] Ashby, M. F. and EAsterling, K. E., Acta Met. 32 (1984) 1935.

[13] LA RoccA, A., Laser Surface Treatment of Metals, Ed. W. Draper and P. Mazzoldi (Martinus Nijhoff Publish.) 521 (1986).

[14] Rosenthal, D., $2^{\mathrm{e}}$ Congrès National des Sciences, Bruxelles, 1277 (1935).

[15] Carslaw, H. S. and Jaeger, J. C., Conduction of Heat in Solids (Clarendon Press) Oxford (1959).
[16] OzıcıK, N., Heat Conduction (Wiley and Sons) New York (1980).

[17] Pittaway, L. G., Brit. J. Appl. Phys. 15 (1964) 967.

[18] Cline, H. E. and Anthony, T. R., J. Appl. Phys. 48 (1977) 3895.

[19] Hsu, S. C., Kou, S. and Mehrabian, R., Met. Trans. B 11B (1980) 29.

[20] Kou, S., Hsu, S. C. and Mehrabian, R., Met. Trans. B 12B (1981) 33.

[21] Sekhar, J. A., Kou, S. and Mehrabian, R., Met. Trans. A 14A (1983) 1169.

[22] SAnders, D. J., Appl. Opt. 23 (1984) 30.

[23] DekumbIS, R., C.T.M.L., E.P.F. Lausanne, Communication Privée.

[24] CoRnillault, J., Le Laser - Principe et Techniques d'Application, Ed. H. Maillet, Tech. Doc. (Lavoisier) Paris, 279 (1985).

[25] LiM, G. C. and Steen, W. M., J. Phys. E 17 (1984) 999.

[26] Dietz, J., Thèse Doctorat, 87 ISAL 0048, Lyon (1987).

[27] Kou, S., Sun, D. K. and Le, Y. P., Metal. Trans. A 14A (1983) 643.

[28] Duley, W. W., Semple, D. J., Morency, J. P. and Gravel, M., Opt. Laser Tech. 11313 (1979).

[29] Ramous, E., Laser Surface Treatment of Metals, Ed. C. W. Draper and P. Mazzoldi (Martinus Nijhoff Publish.) 475 (1986).

[30] Merlin, J. and Dietz, J., Rev. Phys. Appl. 22 (1987) 735 .

[31] Merlin, J. and Dietz, J., $2^{\mathrm{e}}$ partie ; Revue Phys. Appl. 23 (1988) 1807. 